\title{
Experimental investigation on the structure of turbulence in the bottom wave-current boundary layers
}

\author{
Xuan Zhang ${ }^{\text {a,i }}$ and Richard Simons ${ }^{\text {a }}$ \\ a. Department of Civil, Environmental and Geomatic Engineering, University College London, Gower Street, \\ London, WC1E 6BT, UK \\ i. Now working at the Key laboratory of Coastal Disaster and Defense of Ministry of Education, Hohai \\ University.
}

\begin{abstract}
This paper presents insights into the structure of turbulence in combined wavecurrent boundary layers, based on experiments performed in flumes of different scale using Particle Image Velocimetry and hydrogen bubble visualisation. Flow conditions covered a range of wave frequencies, wave amplitudes and mean flow conditions. Results show that the spacing between turbulent streaks varies periodically with the passage of each wave, increasing when the flow accelerates and decreasing when the flow decelerates. A new formula has been put forward, relating the streak spacing variation and the wave-induced orbital displacements. The near-wall flow structure suggests a rhythmic pattern in terms of the velocity gradients across the flume. Waves with higher frequencies and larger amplitudes lead to a greater reduction of mean streak spacing, together with a greater increase of the maximum Reynolds shear stress induced by ejections. These results can be useful for better predictions of the hydrodynamics and sediment transport in combined wave-current flows.
\end{abstract}

Keywords: Wave-current interaction; bottom boundary layer; turbulence; coherent structures; Particle Image Velocimetry; hydrogen bubble visualisation.

\section{Introduction}

The hydrodynamics of the coastal environment is dominated by surface gravity waves and tidal currents. The interaction between such waves and currents is important for engineers concerned with marine energy exploitation, evaluation of coastal erosion, design of coastal structures such as pipelines and tidal turbines, and dispersal of pollutants. Considerable research has been carried out into wave-current interaction (WCI), a term generally referring to the kinematics (velocity field) and dynamics (bed shear stress) induced by the nonlinear interaction between oscillatory waves and a turbulent current. These have been shown to interact in a non-linear way in the near-bottom region (Kemp and Simons, 1982, 1983; Soulsby et al., 1993), leading to a wave-current boundary layer with altered logarithmic velocity profiles and Reynolds shear stresses. The mechanics of WCI have been investigated extensively by analytical approaches (Lundgren, 1972; Grant and Madsen, 1979; Myrhaug, 1982; Fredsøe, 1984; Christoffersen and Jonsson, 1985; Myrhaug and Slaattelid, 1989; You et al., 1991, 1992; You, 1994a, 1994b; Malarkey and Davies, 1998; Umeyama, 2005; 2009; Yuan and Madsen, 2015), laboratory experiments (Bakker and van Doorn, 1978; Van Doorn, 1981; Kemp and Simons, 1982, 1983; Arnskov et al., 1993; Klopman, 1994; Simons et al., 1992, 1994, 2000; Lodahl et al., 1998; Fredsøe et al., 1999; Simons and MacIver, 2001; Umeyama, 2005, 2009, 2011; Carstensen et al., 2010; Yuan and Madsen, 2014, 2015) and numerical methods (Davies et al., 1988; Holmedal et al., 2003; Teles et al., 2012, 2013; Zhang et al., 2014; Zhang et al., 2017).

Coherent vortical structures are the dominant feature in unidirectional turbulent boundary layers, and play crucial roles in carrying turbulent energy and contributing to Reynolds shear stress (Robinson, 1991; Grass, 1971; Grass et al., 1991, 1993; Adrian et al., 2000; Adrian and Liu, 2002; Jiménez, 2013; Dennis, 2015). Low-speed streaks in the viscous sublayer, when lifted up and resulting in bursts or ejections, are a key part of this process. Experimental investigations suggest that sediment particles accumulate along the streaks (Niro and Garcia, 
1996). The whole bursting process provides a theoretical explanation for the path of sediment particles (Sumer and Oguz, 1978; Sumer and Deigaard, 1981).

The characteristics of coherent structures are important for a better evaluation of sediment transport. For instance, the bursting process and turbulent mixing are closely associated with suspension of sediment (Sutherland, 1967; Heathershaw and Thorne, 1985; Nelson et al., 1995; Gyr and Schmid, 1997; Lelouvetel et al., 2009; Keylock et al., 2014). The essential properties of coherent motions, i.e. causing bed shear stress fluctuations, lead to successive saltations of particles. And parting lineation is a sand-bed configuration caused by near-wall streaky patterns. The spanwise spacing is found to be the mean streak spacing (Sleath, 1984). Although there is consensus concerning the nature of coherent structures in turbulent boundary layers, very few investigations have looked at coherent motions in the bottom boundary layer when waves are combined with a turbulent current and the flow is unsteady. Carstensen et al. (2010) performed experiments in an oscillating water tunnel to investigate coherent structures in wave boundary layers and combined wave-current boundary layers. Their work was focused both on the spanwise vortices induced by inflectional-point instability, and on the initiation of turbulent spots which are directly related to the transition and sustaining of a turbulent boundary layer. The present study is closely related to their work, particularly concerning the turbulent spots they observed in combined wave-current boundary layers. The low-speed streaks described in the present study are related to the streaks within the turbulent spots observed by Carstensen et al. (2010). Low-speed streaks normally occur in developed turbulent boundary layers while turbulent spots mark the onset of turbulence. The current Reynolds numbers for the present tests were higher than those included in the work of Carstensen et al. (2010). No coherent transverse vortices were observed in the present tests, which confirms that spanwise vortex tubes are not formed when the current Reynolds numbers are higher than $2.2 \times 10^{4}$ (see Figure 18 of Carstensen et al., 2010). Turbulent spots, which were defined by Carstensen et al. (2010) as 'isolated arrowhead-shaped areas close to the bed in an otherwise laminar boundary layer', were not observed in the present study. Although no data were collected for current Reynolds number higher than $3 \times 10^{4}$, Figure 19 of Carstensen et al. (2010) suggests that turbulent spots should not be observed in the present tests because they were performed in different wave and current Reynolds number regimes. The main objective of the present research is to provide a detailed experimental study on the coherent structures under combined wave and turbulent current conditions, within the fully turbulent current flow regime, which complements the work of Carstensen et al. (2010).

\section{Experimental Set-up}

\subsection{Laboratory flumes}

The first set of experiments were conducted in a low-turbulence flume (Figure 1). This is $6 \mathrm{~m}$ long, $49.3 \mathrm{~cm}$ wide, $27 \mathrm{~cm}$ deep, and equipped with a recirculating system. The flume had glass sidewalls and a glass bed allowing accessibility for photography. The test section was $4.5 \mathrm{~m}$ from the inlet of the flume, and the water depth was kept constant at $0.16 \mathrm{~m}$. This was achieved by a constant head tank with an adjustable weir inside. In order to avoid unwanted turbulence not related to the bottom boundary layer, a basket of glass balls of diameter $19 \mathrm{~mm}$, four stainless steel meshes, and a contraction section were placed in the inlet tank for turbulence suppression. The flow conditioners performed well in smoothing and distributing the flow into the main part of the flume with low turbulence. Incidentally, they also worked well at making the distribution of seeding particles homogeneous. Waves were generated by a plunger-type wave maker. A horizontal permeable metal plate and a sloping sponge layer were used to reduce wave reflections from the outflow weir gate. 
The second set of experiments were carried out in a flume $16 \mathrm{~m}$ long and $0.45 \mathrm{~m}$ wide, with glass sidewalls and bed allowing accessibility for LDV, PIV and photography at the test section. This set of experiments was designed for a different water depth and for the generation of waves with larger amplitudes and larger near-bed velocities (see Tables 1 and 2). The flume had a recirculating system, fed from a header tank $19 \mathrm{~m}$ above and discharging to a sump below floor level. This allowed generation of a steady turbulent current and superposition of waves. The test section where the velocity measurements were made was $8 \mathrm{~m}$ from the inlet. The experimental set-up is shown in Figure 2. To achieve a smooth transition to a combined flow, a conditioner composed of wire meshes was placed at the inlet of the flume. This was found to perform well in suppressing unwanted turbulence created by the inlet geometry. The water depth was kept constant as $\mathrm{h}=0.4 \mathrm{~m}$, and was monitored continuously throughout the experiment. This was maintained by a combination of an adjustable weir gate at the outlet end of the flume and adjustments of the valves. Two pistontype active absorption wave makers were used in the experiments, one positioned at the inlet end to generate waves and the other at the outlet end for reflection absorption. In the present study, sinusoidal waves were generated and propagated in the same direction as the turbulent current. In addition, two permeable horizontal metal plates $2 \mathrm{~m}$ long were placed just below wave trough level at the outlet end of the flume to reduce reflection of high frequency waves (Galleno et al., 2002).

For both sets of experiments, the top surface of the smooth bed at the centre of the flume inlet was taken as the origin of the coordinate system. The $x$-axis was in the streamwise direction, the $y$-axis upward vertically and the $z$-axis representing spanwise direction parallel to the horizontal plane. Based on this coordinate system, $\mathrm{u}, \mathrm{v}, \mathrm{w}$ are the corresponding velocity components.

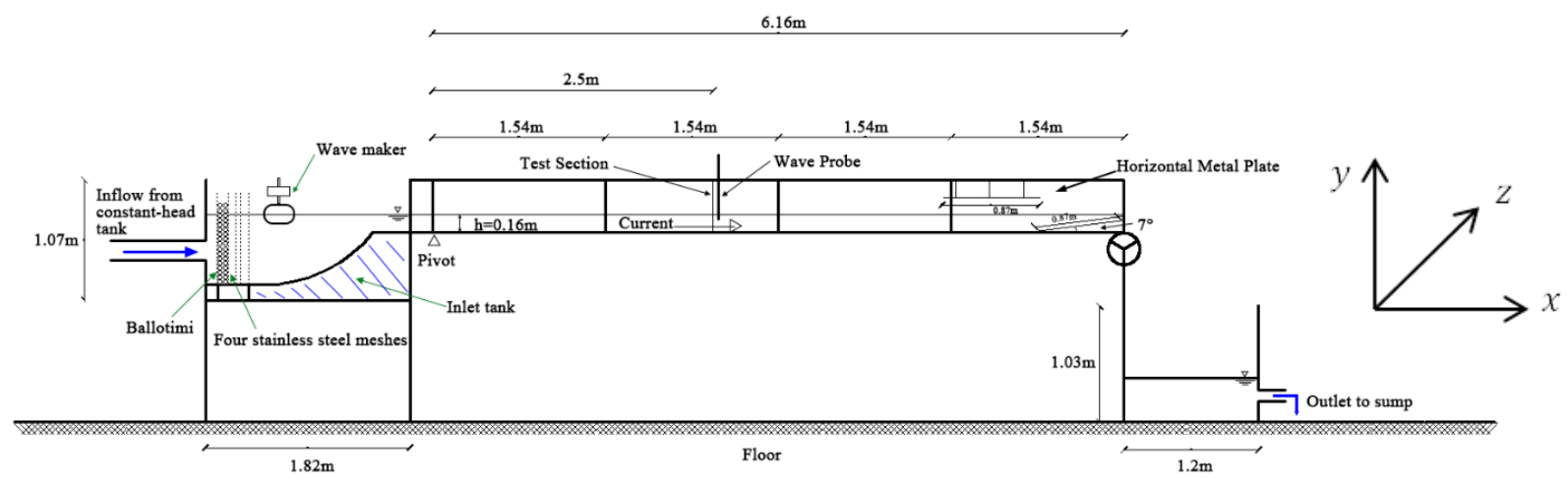

Figure 1. Schematic of the first experimental set-up (side view, not to scale)

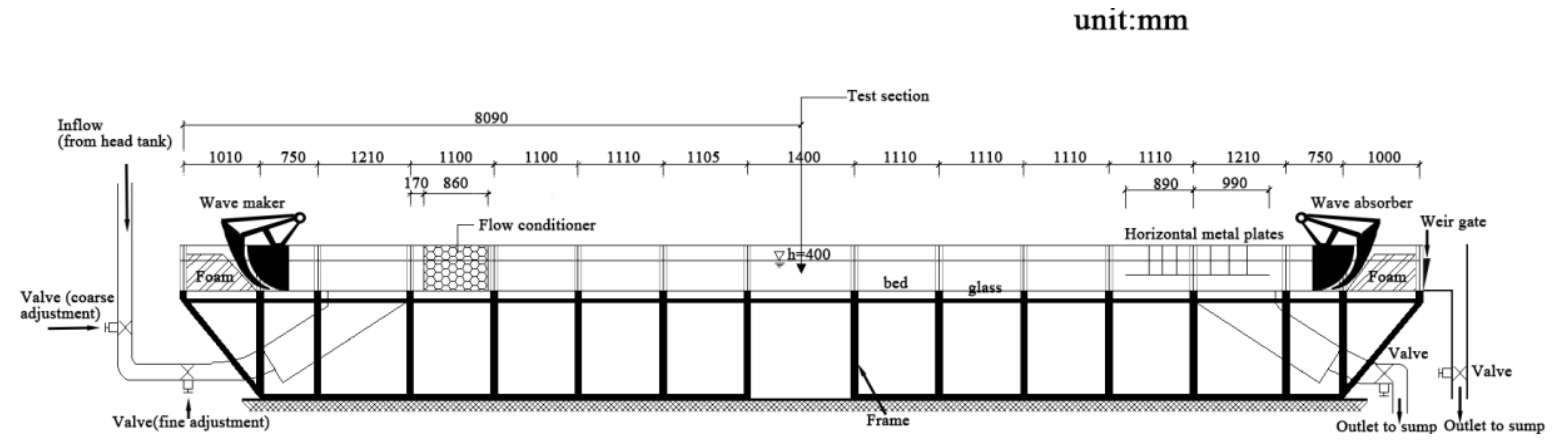

Figure 2. A schematic view of the $16 \mathrm{~m}$ flume, side view. $y \uparrow$

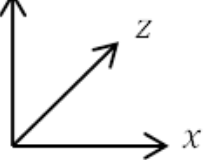




\subsection{Particle Image Velocimetry (PIV)}

The velocities at the test section were measured at the centre of the flume in both sets of experiments, using a two-dimensional Particle Image Velocimetry (PIV) system. A doublepulsed laser with the pulse energy of $150 \mathrm{~mJ}$ was used to produce either a horizontal laser light sheet at $\mathrm{y}=0.8 \mathrm{~mm}$ above the flume bed or a vertical one upwards through the flume bed. This was achieved by positioning a calibration board $(0.8 \mathrm{~mm}$ thick) on the glass bed, and letting the lower edge of the laser touch the calibration board. Previous experiments such as those of Umeyama (2011) used a downwards laser light sheet. However, unsteadiness of the water surface in the form of waves and turbulence means the quality of illumination is steadier and more uniform with an upward light sheet as adopted in the present study. Note that the thickness of the laser light sheet was $1 \mathrm{~mm}$. Therefore, the measurements in the horizontal plane can be influenced by the flow field outside the wave boundary layer. The flow was seeded with hollow microsphere particles $(10 \%$ smaller than $5 \mu \mathrm{m}, 50 \%$ than $10 \mu \mathrm{m}$, $90 \%$ than $21 \mu \mathrm{m}$ and $97 \%$ than $25 \mu \mathrm{m}$ ) by a seeding device placed at the inlet. The illuminated area was photographed by a digital camera with a resolution of $2 \mathrm{~K} \times 2 \mathrm{~K}$ pixels and 16 framesper-second (fps). The camera lens was coupled to a $532 \mathrm{~nm}$ green filter to remove other wavelengths during the tests. A $60 \mathrm{~mm}$ Nikkor lens was used in combination with the camera to obtain the required area of measurements.

Velocity vectors were obtained by a standard commercial type of PIV system (TSI Insight 4G software). A FFT cross-correlation algorithm was adopted to determine the local displacements. A recursive Nyquist grid was applied, with a 50\% overlapping rule for higher spatial resolution (compared with the Nyquist grid) and faster speed (compared with the deformed grid). The size of the grid was set as the default, with a starting spot size of $64 \times 64$ and a final spot size of $32 \times 32$. Post-processing was carried out by a universal median test to detect erroneous vectors. The difference between each velocity vector and the reference vector was normalised by the median of all the differences within a certain neighbour area, and the non-dimensional difference was then compared with the user-defined tolerance. A neighbour area of $3 \times 3$ pixel was adopted, and the median value of all the velocity vectors within this area was used as the reference vector. The interpolated vectors were strictly controlled within $5 \%$ of the whole sample to ensure high-quality data.

\subsection{Hydrogen bubble visualisation}

The hydrogen bubble visualisation technique, based on electrolytic dissociation of water, is useful for both qualitative and quantitative analysis. In the present experiments, the electrical current was supplied by a 50 volt pulse generation box. This can be either a continuous or a pulsed electric current with a wide range of pulsing frequencies. Four copper plates were immersed in the fluid, stuck to the walls, and functioned as the anode. A taut horizontal wire ( $70 \%$ platinum $-30 \%$ iridium alloy, $\mathrm{D}=0.025 \mathrm{~mm}$ ) was positioned at the test section using a supporting system (Figure 3) and submerged in the fluid at the required height. With a negative voltage applied, it formed the cathode. A Continuous Wave Argon Ion Laser was used to give a horizontal laser light sheet. It was adjusted to 1.20 watt to give a stable and high quality illuminated plane with a thickness between $0.1 \mathrm{~mm}$ and $0.25 \mathrm{~mm}$. A camera with a resolution of 1920 pixels $\times 1090$ pixels was used to capture details of the hydrogen bubble tracers from underneath the flume. A video was recorded at 25 frames per second for each test condition. Note that the hydrogen bubble visualisation was only used as an additional tool to investigate streaky patterns and for comparisons with PIV measurements. Taking into account the time required for data processing, this was restricted to use in the small flume. 


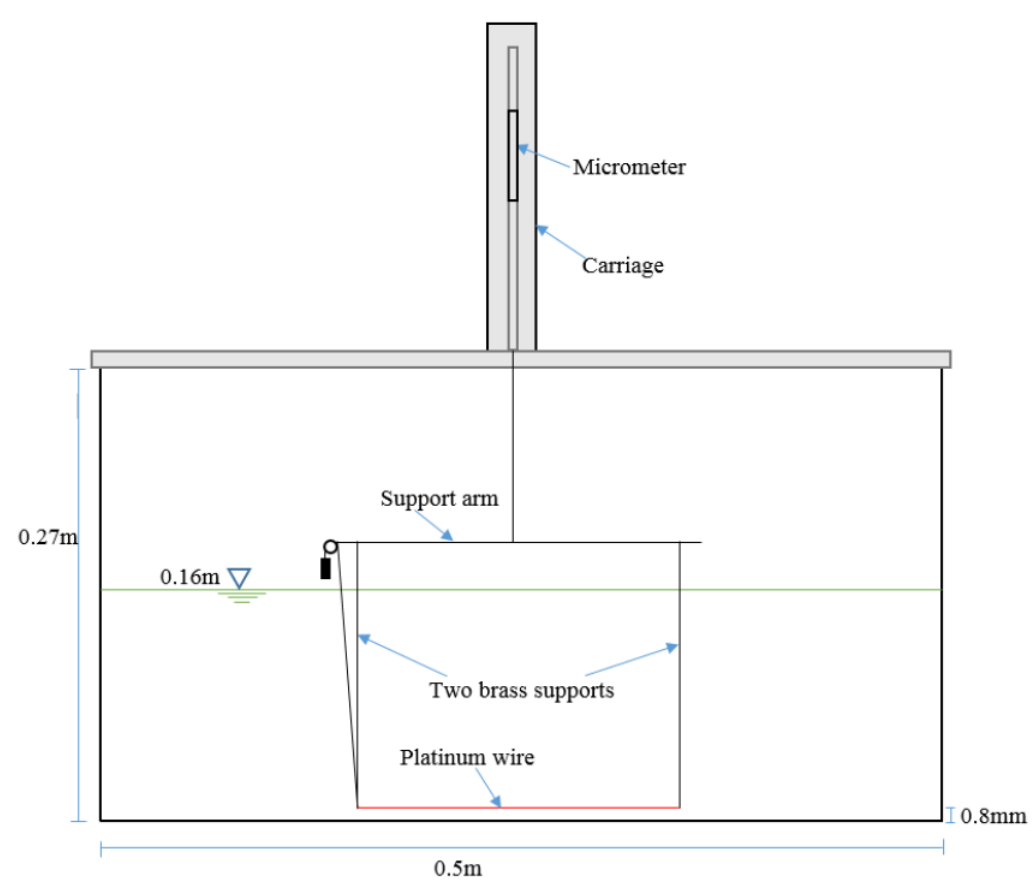

Figure 3. Wire positioning system, mounted at the test section: view looking upstream.

\subsection{Experimental procedures}

The PIV sampling frequency for the tests was set in the range from $4 \mathrm{~Hz}$ to $7.25 \mathrm{~Hz}$, depending on the wave frequency (Tables 1 and 2). Frequencies were chosen to aid ensemble averaging by having an integer number of values of velocity in each wave period. Particular attention should be paid to the sampling frequencies, in order to capture the features of low-speed streaks. Kim et al. (1971) investigated the bursting process using hydrogen-bubble measurements and hot-wire measurements. Curve-fitting results suggested that the time between two consecutive turbulent bursts $T_{B}$ is related to the flow parameters by the expression: $T_{B} \cdot U_{\infty} / \delta=5$, where $U_{\infty}$ is the free-stream velocity and $\delta$ is the boundary layer thickness. Note that even for the lowest sampling frequency $(4 \mathrm{~Hz})$ used here, there will be 8 values of velocity recorded between consecutive bursts using the estimate for $T_{B}$ from Kim et al., ie $d t,=T_{B} / 8$. Smith and Metzler (1983) studied the characteristics of low-speed streaks using a high-speed video system and hydrogen bubble-wire flow visualization. They introduced a parameter $d t^{+}=d t \cdot \frac{u_{*}{ }^{2}}{v}$, where $d t^{+}$and $d t$ are the non-dimensional and the dimensional time between two consecutive measurements respectively. Using this definition in the present investigation, the value of $d t^{+}$was 13 for the lowest sampling frequency $(4 \mathrm{~Hz})$. This value was much smaller than that adopted by Smith and Metzler (1983). On that basis, it was decided that this sampling frequency is enough to capture the features of low-speed streaks. However, it should also be noted that due to the rather large sampling volume, PIV is not capable of capturing all the turbulent motions, compared to other velocity measurement techniques such as LDV. This has already been discussed by Yuan and Madsen (2014), by comparing the Reynolds shear stress to the total shear stress obtained by applying the momentum integral to the measurements.

The mean velocity of the unidirectional current $U_{c}$ was calculated by averaging the mean velocities over depth. The wave orbital velocity amplitude $U_{w}$ was calculated from wave parameters using second-order Stokes wave theory at the bed. The current Reynolds number was defined as $\operatorname{Re}_{c}=U_{c} \cdot h / v$, where $v$ is the kinematic viscosity. The wave Reynolds 
number $\operatorname{Re}_{w}$ was defined as $\operatorname{Re}_{w}=U_{w} \cdot A_{w} / v$, where $A_{w}$ is the wave semi-orbital excursion $A_{w}=U_{w} \cdot T / 2 \pi$ at the bed. It should be noted here that the test names include the wavemaker stroke ' $\mathrm{A}$ '. This parameter is different from the wave surface amplitude, which is half the wave height for linear waves. A wave probe was positioned close to the test section but not interfering with the PIV measurements, to measure the free surface elevations. Collection of the wave data was triggered by the initial firing of the first laser when acquiring each set of image pairs. There was a settling period of 30-60 minutes before starting experiments; data were collected 50s after wave generation was started.

For the experiments in the low-turbulence flume, 600 and 380 pairs of images were captured sequentially for the $\mathrm{u}-\mathrm{w}$ measurements and $\mathrm{u}-\mathrm{v}$ measurements respectively. The key parameter, time $d t$ between frame $\mathrm{A}$ and frame $\mathrm{B}$, was set as $d t=800 \mu \mathrm{s}$ and $d t=2600 \mu \mathrm{s}$, respectively. Each pair of images covered an area $76 \mathrm{~mm} \times 76 \mathrm{~mm}$ (horizontal plane) and $195 \mathrm{~mm} \times 195 \mathrm{~mm}$ (vertical plane). Processing of the images and the calibrations led to a spatial resolution of $0.6 \mathrm{~mm}$ in the horizontal plane and $1.4 \mathrm{~mm}$ in the vertical plane. Details of the processing algorithms are included in section 2.2. It should be noted that the calibrations were performed three times and the average value was used to increase accuracy.

For measurements in the $16 \mathrm{~m}$ flume, a sequence of 770 image pairs was recorded. The time was set as $d t=1000 \mu \mathrm{s}$ (horizontal plane) and $d t=3000 \mu$ s (vertical plane). Spatial resolution was similar to that in the low-turbulence flume.

Table 1. Flow conditions for experiments conducted in the low-turbulence flume, water depth $h=160 \mathrm{~mm}$.

\begin{tabular}{|c|c|c|c|c|c|c|c|c|c|}
\hline Test Name & $\begin{array}{l}\text { Flow } \\
\text { Type }\end{array}$ & $\mathrm{T}(\mathrm{s})$ & $\begin{array}{c}\mathrm{H} \\
(\mathrm{mm})\end{array}$ & $\begin{array}{c}\text { PIV sampling } \\
\text { frequency } \\
\text { f(Hz) }\end{array}$ & $\begin{array}{c}U_{c} \\
(\mathrm{~m} / \mathrm{s})\end{array}$ & $\begin{array}{c}U_{w} \\
(\mathrm{~m} / \mathrm{s})\end{array}$ & $R e_{c}$ & $R e_{w}$ & $\begin{array}{l}U_{c w} \\
=U_{c} /\left(U_{c}\right. \\
\left.+U_{m}\right)\end{array}$ \\
\hline $\mathrm{CA}$ & $\begin{array}{l}\text { Current- } \\
\text { only }\end{array}$ & -- & -- & 7.25 & \multirow{16}{*}{0.200} & -- & \multirow{16}{*}{32000} & -- & 1.00 \\
\hline WCAT1.11sA12mm & \multirow{3}{*}{$\begin{array}{l}\text { Wave- } \\
\text { current } \\
\text { condition }\end{array}$} & \multirow{3}{*}{1.11} & 9 & \multirow{3}{*}{4.50} & & 0.029 & & 154 & 0.87 \\
\hline WCAT1.11sA14mm & & & 11 & & & 0.035 & & 221 & 0.85 \\
\hline WCAT1.11sA18mm & & & 14 & & & 0.046 & & 378 & 0.81 \\
\hline WCAT1.25sA14mm & \multirow{4}{*}{$\begin{array}{l}\text { Wave- } \\
\text { current } \\
\text { condition }\end{array}$} & \multirow{4}{*}{1.25} & 11 & \multirow{4}{*}{4.00} & & 0.039 & & 314 & 0.84 \\
\hline WCAT1.25sA16mm & & & 13 & & & 0.045 & & 405 & 0.82 \\
\hline WCAT1.25sA18mm & & & 14 & & & 0.049 & & 482 & 0.80 \\
\hline WCAT $1.25 \mathrm{sA} 20 \mathrm{~mm}$ & & & 16 & & & 0.056 & & 634 & 0.78 \\
\hline WCAT1.38sA12mm & \multirow{5}{*}{$\begin{array}{l}\text { Wave- } \\
\text { current } \\
\text { condition }\end{array}$} & \multirow{5}{*}{1.38} & 11 & \multirow{5}{*}{7.25} & & 0.042 & & 397 & 0.83 \\
\hline WCAT1.38sA14mm & & & 13 & & & 0.049 & & 532 & 0.80 \\
\hline WCAT1.38sA16mm & & & 15 & & & 0.055 & & 691 & 0.78 \\
\hline WCAT1.38sA18mm & & & 15 & & & 0.058 & & 747 & 0.78 \\
\hline WCAT1.38sA20mm & & & 18 & & & 0.066 & & 986 & 0.75 \\
\hline WCAT1.67sA14mm & \multirow{3}{*}{$\begin{array}{l}\text { Wave- } \\
\text { current } \\
\text { condition }\end{array}$} & \multirow{3}{*}{1.67} & 11 & \multirow{3}{*}{6.00} & & 0.042 & & 482 & 0.83 \\
\hline WCAT1.67sA16mm & & & 12 & & & 0.048 & & 624 & 0.81 \\
\hline WCAT1.67sA18mm & & & 14 & & & 0.055 & & 806 & 0.78 \\
\hline
\end{tabular}


Table 2. Flow conditions for experiments conducted in the $16 \mathrm{~m}$ flume, water depth $h=$ $400 \mathrm{~mm}$.

\begin{tabular}{|c|c|c|c|c|c|c|c|c|c|}
\hline Test Name & Flow Type & $\begin{array}{l}\mathrm{T} \\
(\mathrm{s})\end{array}$ & $\begin{array}{c}\mathrm{H} \\
(\mathrm{mm})\end{array}$ & $\begin{array}{c}\text { PIV } \\
\text { sampling } \\
\text { frequency } \\
\mathrm{f}(\mathrm{Hz})\end{array}$ & $\begin{array}{c}U_{c} \\
(\mathrm{~m} / \mathrm{s})\end{array}$ & $\begin{array}{c}U_{w} \\
(\mathrm{~m} / \mathrm{s})\end{array}$ & $R e_{c}$ & $R e_{w}$ & $U_{c w}$ \\
\hline CAA & Current-only & & & 7.0 & \multirow{7}{*}{0.175} & -- & \multirow{7}{*}{71200} & -- & 1.00 \\
\hline WCAAT2sA70mm & $\begin{array}{l}\text { Wave-current } \\
\text { condition }\end{array}$ & 2.00 & 83 & 5.0 & & 0.204 & & 13546 & 0.46 \\
\hline WCAAT1.67sA120mm & $\begin{array}{c}\text { Wave-current } \\
\text { condition }\end{array}$ & 1.67 & 120 & 6.0 & & 0.266 & & 23194 & 0.40 \\
\hline WCAAT1.43sA120mm & $\begin{array}{l}\text { Wave-current } \\
\text { condition }\end{array}$ & 1.43 & 138 & 7.0 & & 0.269 & & 16573 & 0.39 \\
\hline WCAAT $1.25 \mathrm{sA} 100 \mathrm{~mm}$ & $\begin{array}{c}\text { Wave-current } \\
\text { condition }\end{array}$ & 1.25 & 110 & 4.0 & & 0.184 & & 7119 & 0.49 \\
\hline WCAAT1.11sA86mm & $\begin{array}{l}\text { Wave-current } \\
\text { condition }\end{array}$ & 1.11 & 83 & 4.5 & & 0.117 & & 3138 & 0.60 \\
\hline WCAAT1sA70mm & $\begin{array}{l}\text { Wave-current } \\
\text { condition }\end{array}$ & 1.00 & 52 & 5.0 & & 0.061 & & 1033 & 0.74 \\
\hline
\end{tabular}

\section{Data Analysis methods}

\subsection{Turbulent fluctuations}

In a unidirectional current, instantaneous velocities $(u)$ are made up of a time-averaged component $(\bar{u})$ and turbulent fluctuations $\left(u^{\prime}\right)$, while in a combined wave-current flow they include an additional wave-induced periodic component ( $\tilde{u})$ (Nielsen, 1992). In general, for a velocity component $M$, this is expressed by the following equation:

$$
\begin{aligned}
& M=\bar{M}+\widetilde{M}+M^{\prime} \\
& \langle M\rangle=\frac{1}{J} \sum_{j=0}^{J-1} M(t+j \cdot T), \quad 0 \leq t<T \\
& \widetilde{M}(t)=\langle M\rangle-\bar{M}, \quad 0<t<T
\end{aligned}
$$

where $\overline{\mathrm{M}}$ is the time-average of $M$ over the samples, $\widetilde{\mathrm{M}}$ is the periodic component, $\langle\mathrm{M}\rangle$ is the ensemble-average of $M, \mathrm{M}^{\prime}$ is turbulent component, $T$ is wave period, $J$ is the number of wave cycles for ensemble-averaging.

The determination of ensemble-averaged velocities in the present study differed slightly from previous methods for a single point measurement because of the PIV technique adopted. Here, ensemble-averaged velocities were calculated using three-point spatial-averaging. This provided high frequency smoothing and increased the number of independent data points included in the ensemble-average by a factor of 3 . Since this was performed only within $4.5 \mathrm{~mm}$, which is small compared to the wavelength, there was a negligible difference between the velocity components within the adjacent three points used for spatial-averaging. Therefore, the number of cycles used for ensemble-averaging was in line with those adopted in previous studies and was enough to produce statistically reliable data. Note that the threepoint spatial averaging method was only used for $\mathrm{u}-\mathrm{v}$ measurements in combined wavecurrent flows to obtain wave-induced periodic velocities. This should be differentiated from the spatial-averaging over 71 points as shown in the next section, which was used to determine the time-averaged mean velocity and Reynolds shear stress distributions.

\subsection{Reynolds shear stress and mean velocity profiles}

Reynolds shear stress were determined by time-averaging and spatial-averaging. This is valid for the turbulent current flow with and without waves superimposed, with turbulent fluctuations determined from the ensemble-averaging procedure; 


$$
\overline{\tau_{R e y}}(y)=-\frac{1}{71} \sum_{i_{1}=1}^{71} \frac{1}{N} \sum_{n=0}^{N-1} \rho \cdot u^{\prime}\left(x_{i 1}, y, n \cdot d t\right) \cdot v^{\prime}\left(x_{i 1}, y, n \cdot d t\right)
$$

where $\overline{\tau_{R e y}}(y)$ represents the vertical distribution of Reynolds shear stress, $\rho$ the water density.

The spatial-averaging was performed over the centre of each image, for 71 points in the $x$ direction, so as to eliminate influences from the edges of PIV images. Differences in the longitudinal distribution of Reynolds shear stress are also negligibly small, and therefore spatial averaging in the $x$ direction of each PIV image was adopted in the present work.

The vertical distribution of mean velocity $\bar{U}(y)$ was obtained following the same procedure, by time-averaging and spatial-averaging:

$$
\bar{U}(y)=\frac{1}{71} \sum_{i_{1}=1}^{71} \frac{1}{N} \sum_{n=0}^{N-1} u\left(x_{i_{1}}, y, n \cdot d t\right)
$$

\subsection{Spatial structure of turbulent low-speed streaks}

Auto-correlation of streamwise velocities was applied to detect periodicity of turbulent structures and to determine the spacing between low-speed streaks. The instantaneous twopoint auto-correlation coefficient can be calculated from (6), using the definition given by Pope (2000):

where

$$
R\left(x_{i_{1}}, d z, t\right)=\frac{\frac{1}{56} \cdot \sum_{i_{3}=1}^{56} u\left(x_{i_{1}}, z_{i_{3}}, t\right)^{\prime \prime} \cdot u\left(x_{i_{1}}, z_{i_{3}}+d z, t\right)^{\prime \prime}}{\frac{1}{56} \cdot \sum_{i_{3}=1}^{56} u\left(x_{i_{1}}, z_{i_{3}}, t\right)^{\prime \prime} \cdot u\left(x_{i_{1}}, z_{i_{3}}, t\right) \prime \prime}
$$

$$
\begin{aligned}
& u\left(x_{i_{1}}, z_{i_{3}}, t\right)^{\prime \prime}=u\left(x_{i_{1}}, z_{i_{3}}, t\right)-U\left(x_{i_{1}}, t\right) \\
& d z=r \cdot \Delta, \quad r=0,1,2, \ldots, 55 \\
& U\left(x_{i_{1}}, t\right)=\frac{1}{112} \sum_{i_{3}=1}^{112} u\left(x_{i_{1}}, z_{i_{3}}, t\right)
\end{aligned}
$$

and $\Delta$ is the grid size in the spanwise direction $(\Delta=0.6 \mathrm{~mm}), u(z)^{\prime \prime}$ is the velocity fluctuation in the spanwise direction, $I_{3}$ is the total number of grid points in $z$ direction $\left(I_{3}=127\right)$, $\mathrm{U}\left(\mathrm{x}_{i_{1}}, \mathrm{t}\right)$ is the instantaneous streamwise velocity averaged across the $z$-axis and therefore is only a function of streamwise position $\mathrm{x}_{i_{1}}$ and time $t$.

The instantaneous integral spatial scale was determined by analogy to the integral time scale in turbulence theories:

$$
\text { I.S.S }\left(x_{i_{1}}, t\right)=\int_{0}^{z_{0}} R\left(x_{i_{1}}, d z, t\right) d z
$$

where $z_{0}$ is the first zero-crossing point of the auto-correlation coefficient distributions $R\left(\mathrm{x}_{i_{1}}, \mathrm{dz}, \mathrm{t}\right)$.

The instantaneous streak spacing at a fixed $x$ position $\lambda\left(x_{i_{1}}, t\right)$ was then obtained as the value of $d z$ corresponding to the first peak of $\mathrm{R}\left(x_{i_{1}}, d z, t\right)$. For simplicity, the instantaneous value of streak spacing is denoted as $\lambda(t)$.

The time-averaged mean streak spacing $\bar{\lambda}$ was then obtained by averaging over all samples:

$$
\bar{\lambda}=\frac{1}{N} \sum \lambda(t)
$$

Statistical analysis was performed to investigate the probability density distribution of streak spacing, in terms of standard deviation $\sigma_{\lambda}$, coefficient of variation $\psi_{\lambda}$, skewness $S_{\lambda}$, and flatness $F_{\lambda}$. Definitions of these parameters can be found in Pope (2000).

In the present study, the lognormal distribution and the Burr distribution were both applied. The Burr distribution, originally proposed by Burr (1942), is more flexible and can represent a wider range of distribution shapes. It was originally controlled by two parameters but later developed by Tadikamalla (1980) with an additional parameter. The probability density function is given by: 


$$
P(\lambda(t))=\frac{\frac{\xi \Omega}{r}\left[\frac{\lambda(t)}{\gamma}\right]^{\Omega-1}}{\left\{1+\left[\frac{\lambda(t)}{\gamma}\right]^{\Omega}\right\}^{\xi+1}}
$$

where $\xi$ and $\Omega$ are the shape parameters and $Y$ is the scale parameter.

The ensemble-averaged streak spacing was determined in a similar way to ensembleaveraged velocities, in order to quantify the evolution of streak spacing within one wave cycle:

$$
\langle\lambda\rangle(t)=\frac{1}{J} \sum_{j=0}^{J-1} \lambda(t+j \cdot T), \quad 0<t<T
$$

The periodic streak spacing $\tilde{\lambda}$ was then determined by subtracting the time-averaged streak spacing from the ensemble-averaged streak spacing, in analogy to periodic velocity component:

$$
\tilde{\lambda}(t)=\frac{1}{J} \sum_{j=0}^{J-1} \lambda(t+j \cdot T)-\bar{\lambda}, \quad 0<t<T
$$

\subsection{Quadrant analysis of Reynolds shear stress}

Reynolds shear stress can be generated from four quadrant events: Q1 events $\left(u^{\prime}>0 \& v^{\prime}>\right.$ $0)$, Q2 events $\left(u^{\prime}<0 \& v^{\prime}>0\right)$, Q3 events $\left(u^{\prime}<0 \& v^{\prime}<0\right)$, and Q4 events $\left(u^{\prime}>0 \& v^{\prime}<\right.$ $0)$. The definition of quadrant analysis originally proposed by Lu and Willmarth (1973, pp. 14) was adopted to investigate the contributions from the four events to Reynolds shear stress. According to this definition, Q1 events are alternatively known as outward interactions, Q2 events as ejections, Q3 events as wallward interactions, and Q4 events as sweeps. The spatial-averaging procedure was then performed again to quantify the magnitude of Reynolds shear stress contributed from each quadrant event $\left(\overline{\tau_{R e y_{Q}}}\right)$. This is valid for a turbulent current flow with and without waves added.

\subsection{Data Analysis for hydrogen bubble experiments}

The main purpose of the hydrogen bubble experiments was to provide additional qualitative information of flow patterns in the near-bed region, for a turbulent current with and without waves superimposed. Quantitative analysis was performed for the turbulent current flow without the waves, based on the sequence of 75 frames analysed with a duration of $3 \mathrm{~s}$. The first step was to obtain the whole sequence of instantaneous frames from the video records. Image analysis was then performed to improve the image quality. The processed image was then used to obtain the coordinates of the timeline for each image, so as to determine the displacements of the bubbles. Having obtained the instantaneous spanwise distribution of streamwise velocity $u(x, z, t)$, the instantaneous spacing between low-speed streaks was quantified using the same procedure as detailed in section 3.3.

\section{Experimental results}

\subsection{Unidirectional Currents without waves}

Results of mean velocities and Reynolds shear stress demonstrate that the turbulent currents generated in both flumes conform to classical theory and that the number of independent samples was adequate to produce statistically reliable results. Bed shear stress, shear velocities $u_{* \mathrm{c}}$ and boundary layer thickness were determined from Reynolds shear stress distributions. Logarithmic regions were observed in the bottom $30 \%$ of the boundary layer, agreeing with Adrian et al. (2000). The von Kármán constant was obtained by applying the logarithmic law of the wall. The log-law constant was taken as 5.2 (Pope, 2000), and the first point used for curve-fittings was chosen in the logarithmic region $\left(y^{+}>30\right)$. This leads to the values: $\kappa=0.3$ for the low-turbulence flume and 0.37 for the $16 \mathrm{~m}$ flume.

High-speed and low-speed streaks were observed in the viscous sublayer. The former were relatively wider than the latter, agreeing with Robinson (1991). Low-speed streaks were 
elongated in the streamwise direction and tended to meander in the spanwise direction. A typical example is given in Figure 4. Regions of high-speed and low-speed streaks are highlighted by red and black ellipses, and denoted as 'HSS' and 'LSS' respectively. Applying Taylor's hypothesis (Taylor, 1938), Figure 4 (a) to (c) illustrate that these high-speed and low-speed streaks all move downstream approximately at the local mean velocity.

Results of non-dimensional streak spacing are tabulated in Table 3, together with values provided by previous experiments in the literature. The present values are close to but slightly higher than the values in the literature.

The probability density functions of streak spacing (Figure 5) show that a Burr distribution fits better than a lognormal one. This is different from previous observations (Nakagawa and Nezu, 1981; Smith and Metzler, 1983; and Mansour-Tehrani, 1992). The better performance of a Burr distribution is reflected in the statistical parameters.

Table 3. Comparison of non-dimensional mean streak spacing $\lambda^{+}$with literature.

\begin{tabular}{|c|c|}
\hline Investigators & $\lambda^{+}=\bar{\lambda} \cdot u_{* c} / v$ \\
\hline $\begin{array}{c}\text { Present study in the low-turbulence } \\
\text { flume, results from PIV measurements }\end{array}$ & 118 \\
\hline $\begin{array}{c}\text { Present study in the low-turbulence } \\
\text { flume, results from hydrogen bubble } \\
\text { visualisations }\end{array}$ & 111 \\
\hline $\begin{array}{c}\text { Present study in the 16m flume, results } \\
\text { from PIV measurements }\end{array}$ & Ranges from 91 to 106 \\
\hline Schraub and Kline (1965) & Ranges from 91 to 106 (visual counting \\
\hline Kline et al. (1967) & Ranges from 89 to 151 \\
\hline Gupta et al. (1971) & Ranges from 105 to 107 \\
\hline Lee et al. (1974) & Ranges from 79 to 93 \\
\hline Achia and Thompson (1976) & Ranges from 88 to 108 \\
\hline Oldaker and Tiederman (1977) & Ranges from 100 to 113 \\
\hline Nakagawa \& Nezu (1981) & Ranges from 87 to 104 \\
\hline Smith and Metzler (1983) & 101 \\
\hline Grass et al. (1991) & 101 \\
\hline Mansour-Tehrani (1992) & \\
\hline Lagraa et al. (2004) & Ran 110 \\
\hline
\end{tabular}



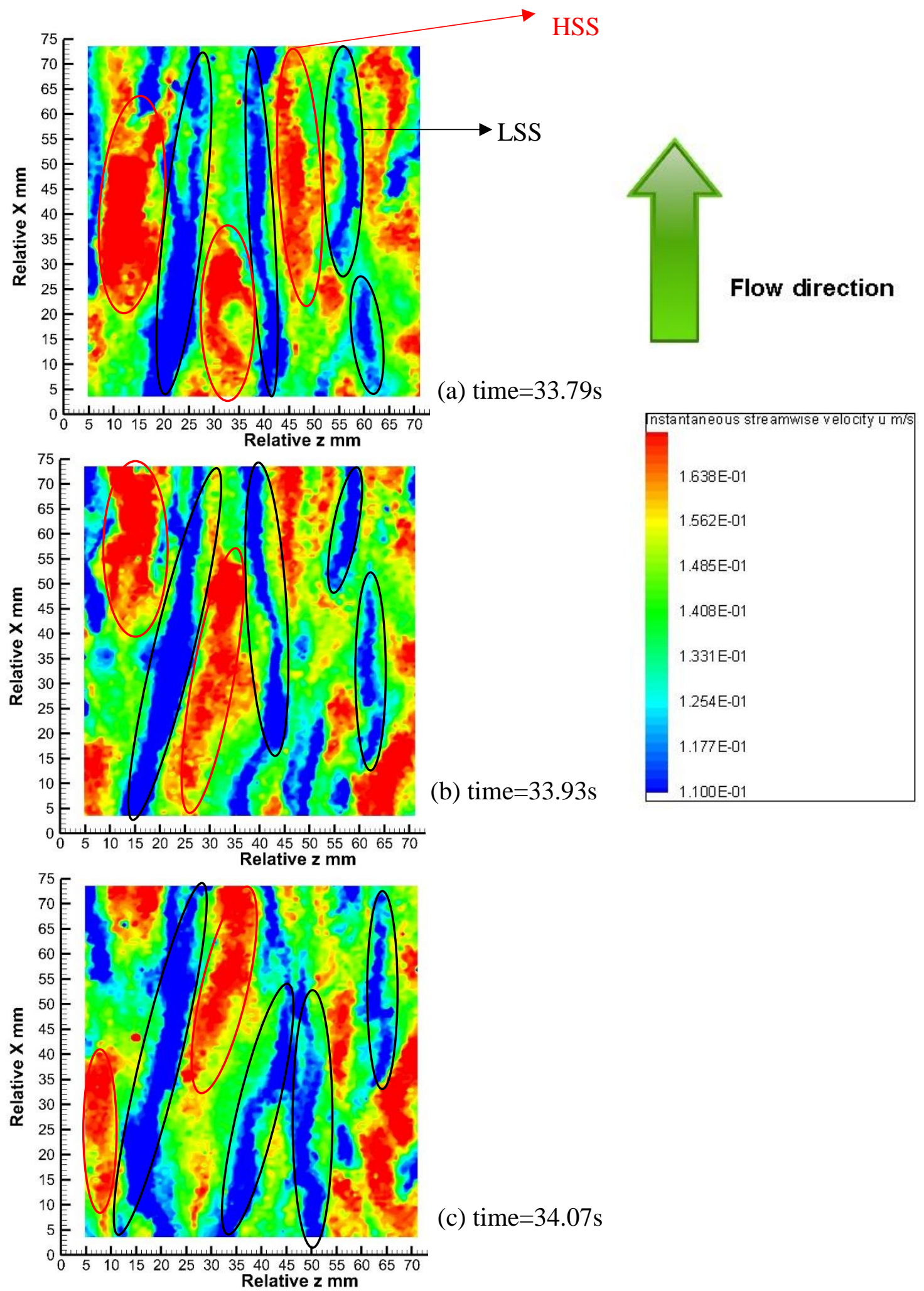

Figure 4. Time histories of instantaneous streamwise velocity contours from PIV measurements, current-alone test in the low-turbulence flume, plan view, $y=0.8 \mathrm{~mm}\left(\mathrm{y}^{+}=6\right)$, above the bed. 


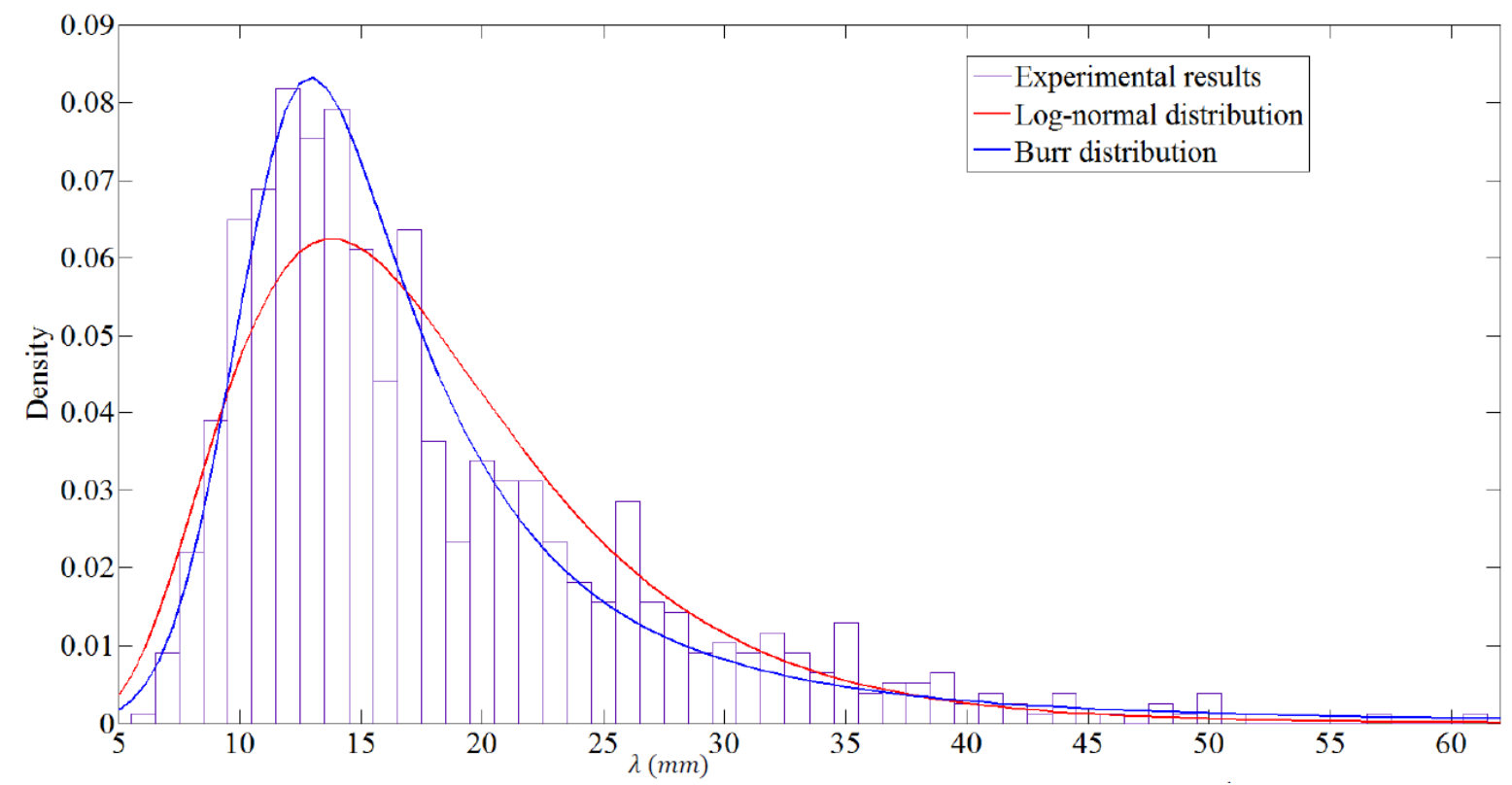

Figure 5. Probability density functions of streak spacing at $\mathrm{y}=0.8 \mathrm{~mm}\left(\mathrm{y}^{+}=6\right)$, unidirectional turbulent current, PIV measurements in the $16 \mathrm{~m}$ flume.

\subsection{Combined wave-current flows}

\subsubsection{Mean velocity profiles and Reynolds shear stress}

As can be seen from Tables 1 and 2, the tests cover conditions in both the current-dominated and wave-dominated regimes. For most of the conditions tested, the wave-current parameter $U_{c w}>0.5$. Such combined wave-current flows lie in the current-dominated regime and no re-laminarisation would occur (Lodahl et al., 1998). However, it should be noted that relaminarisation may happen in some tests in the $16 \mathrm{~m}$ flume, since the flow is wave-dominated and the wave boundary layer is in the laminar regime.

Mean velocity profiles are shown in Figure 6. The levels of the wave troughs are highlighted using a horizontal line. Note that velocities were not measured above the trough levels. It should also be noted that velocities in the upper water column were not measured in the $16 \mathrm{~m}$ flume since they are not relevant to the present investigation. Therefore, no trough levels are highlighted in Figure 6(b). Mean velocities increase near the bed when waves are added. In the upper flow region, however, the $16 \mathrm{~m}$ flume tests show a decrease while the lowturbulence flume tests remain similar to the current-only test. The observations in the $16 \mathrm{~m}$ flume agree well with earlier studies on wave-current interaction (e.g., Kemp and Simons, 1982; Klopman, 1994; Umeyama, 2005; Umeyama, 2009). Results for the other flume are different from classical wave-current interaction. The fundamental difference between the two flume types leads to the increase in the depth-averaged mean velocities observed in the low-turbulence flume. In an open channel, discharge over the weir varies with head and hence increases when the crest passes. No return flow is possible during the wave trough. In contrast, in a closed wave flume such as the $16 \mathrm{~m}$ flume, the flux goes through valves and pipes and there are far less immediate responses to the varying head created by the wave crest. The speed of the return flow predicted by Kim (1984), which is induced to balance the flux due to the Stokes drift and can be assumed uniform and steady (Kim, 1984; Ramsden and Nath, 1989), is similar to the increase in the depth-averaged mean velocities observed when waves are added. This supports the suggestion that increased depth-averaged velocities can be attributed to the absence of a return flow.

Comparisons between tests with the same amplitude and different periods indicate higher velocities near the bed when shorter waves are superimposed. The boundary layer thickness 
is reduced to $20 \mathrm{~mm}$ [Figure 6 (a)]. This reduction is confirmed by the Reynolds shear stress distributions shown later. Figure 6 (b) shows the same trend in the $16 \mathrm{~m}$ flume, as evidenced by comparing the case with wave periods of $1 \mathrm{~s}$ and $2 \mathrm{~s}$ or of $1.43 \mathrm{~s}$ and $1.67 \mathrm{~s}$, although the reduction of boundary layer thickness is not as significant as in the other flume.

The influence of wave amplitude $A$ and period $T$ as observed from the present investigation is consistent with previous research. Since wave-induced bed shear stress is proportional to wave amplitude and frequency (Nielsen, 1992) for a laminar wave bottom boundary layer, it is expected that waves with larger $A$ and shorter $T$ lead to increased wave-induced bed shear stress, more reduction of boundary layer thickness and hence larger increase of bed shear stress.

Reynolds shear stress distributions show that waves with shorter periods and larger amplitudes lead to greater reductions of boundary layer thickness and hence greater shear stress. A typical example is given in Figure 7, and is consistent with mean velocities.
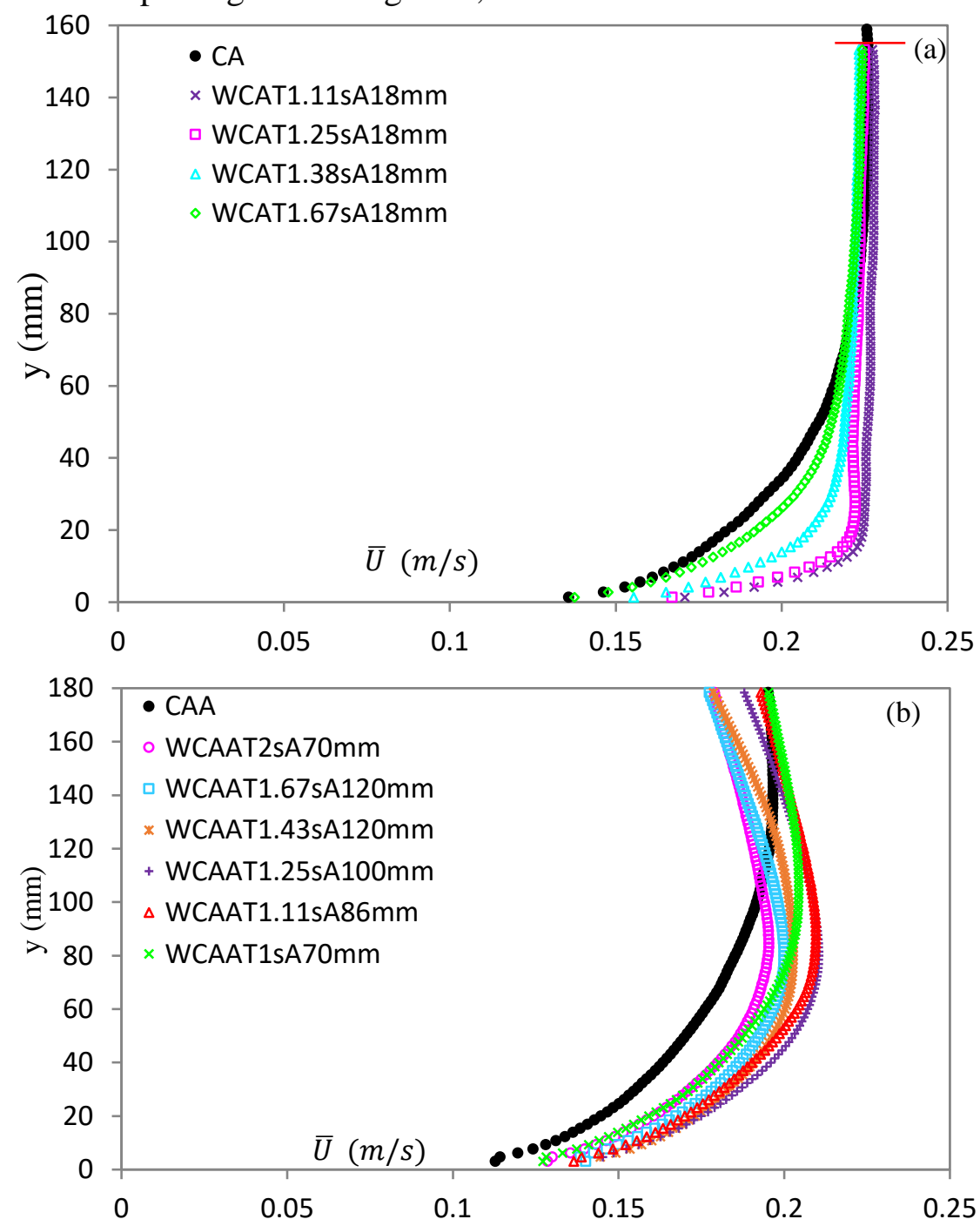

Figure 6. Mean velocity profiles of turbulent currents with and without waves superimposed: (a) tests in the low-turbulence flume, varying period, wave amplitudes fixed at $A=18 \mathrm{~mm}$;

(b) tests in the $16 \mathrm{~m}$ flume. 


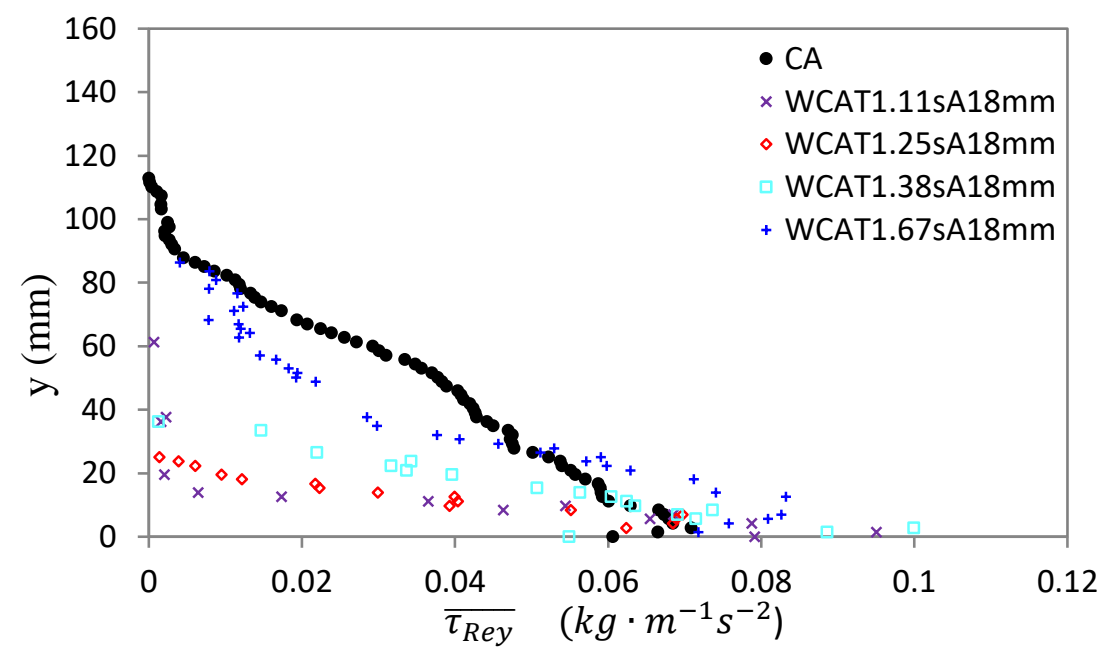

Figure 7. Reynolds shear stress of turbulent currents with and without waves superimposed. Tests in the low-turbulence flume, varying period, wave amplitudes fixed at $A=18 \mathrm{~mm}$.

\subsubsection{Coherent structures: streaky patterns}

Values of the mean streak spacing are tabulated in Tables 4 and 5 for turbulent currents with and without waves superimposed in the two flumes. Although values of $\bar{\lambda}$ are very similar with and without waves superimposed, a small but consistent decrease is observed when waves with increasing amplitudes are added. This can be seen by comparing the conditions of waves with the same period and increasing amplitudes in Table 4 and, for waves with $\mathrm{T}=1.38 \mathrm{~s}$, in Figure 8. The decrease of mean streak spacing is observed to be less than $3 \mathrm{~mm}$ (13\%), compared to the current-alone test. This agrees qualitatively with the increase of shear velocity and reduction of boundary layer thickness, since the streak spacing reflects the scale of streamwise vortices and hence turbulent boundary layer thickness.

Waves with shorter periods lead to a consistent decrease of $\bar{\lambda}$. Results suggest a decrease of $5 \mathrm{~mm}(36 \%)$, as observed from waves with periods of $1.11 \mathrm{~s}$ and $1.67 \mathrm{~s}$ added in the lowturbulence flume (Table 4). Similarly, results from the $16 \mathrm{~m}$ flume show a decrease of $2 \mathrm{~mm}$ (11\%), when comparing waves with periods of $1.11 \mathrm{~s}$ and $1.67 \mathrm{~s}$ (Table 5).

However, the decrease of $\bar{\lambda}$ is not of the same order as that of boundary layer thickness reduction. These results suggest that the well-accepted range of $\lambda^{+}$for unidirectional currents may not be applicable when waves are added. Results of $\lambda^{+}$confirm that $\lambda^{+}$is higher for combined wave-current flows. Results (Figure 9) indicate that $\lambda^{+}=\frac{\bar{\lambda} \cdot u_{* \mathrm{wc}}}{v}=140$ holds for combined wave-current boundary layers at both laboratory scales tested. For low $\operatorname{Re}_{\mathrm{w}}$ it increases more significantly. This is probably attributable to relaminarization. The very strong interaction between the oscillations and the turbulence structures may also contribute to the increase of $\lambda^{+}$(Ramaprian and Tu, 1983). Note that $u_{* \text { wc }}$ represents the shear velocities in combined wave-current flows. Values were determined from the Reynolds shear stress distributions (section 4.2.1), assuming a linear distribution within the boundary layers. See Tables 4 and 5 for the results. 
Table 4. Mean streak spacing $\bar{\lambda}$, tests conducted in the low-turbulence flume.

\begin{tabular}{|c|c|c|c|c|c|c|c|c|c|}
\hline Case Conditions & $\bar{\lambda}(\mathrm{mm})$ & $\begin{array}{c}u_{* \mathrm{c}} \text { or } \\
u_{* \mathrm{wc}} \\
(\mathrm{m} / \mathrm{s})\end{array}$ & $\lambda^{+}$ & $y^{+}$ & Case Conditions & $\bar{\lambda}(\mathrm{mm})$ & $\begin{array}{c}u_{* \mathrm{wc}} \\
(\mathrm{m} / \mathrm{s})\end{array}$ & $\lambda^{+}$ & $y^{+}$ \\
\hline CA & 16 & 0.008 & 118 & 6 & WCAT1.38sA20mm & 15 & 0.010 & 143 & 8 \\
\hline WCAT1.11sA12mm & 16 & 0.010 & 152 & 8 & WCAT1.25sA14mm & 17 & 0.010 & 160 & 8 \\
\hline WCAT1.11sA14mm & 15 & 0.010 & 143 & 8 & WCAT1.25sA16mm & 15 & 0.011 & 157 & 8 \\
\hline WCAT1.11sA18mm & 14 & 0.012 & 155 & 9 & WCAT1.25sA18mm & 14 & 0.011 & 141 & 8 \\
\hline WCAT1.38sA12mm & 17 & 0.010 & 157 & 7 & WCAT1.25sA20mm & 14 & 0.011 & 148 & 8 \\
\hline WCAT1.38sA14mm & 17 & 0.010 & 157 & 7 & WCAT1.67sA14mm & 20 & 0.010 & 185 & 7 \\
\hline WCAT1.38sA16mm & 17 & 0.010 & 157 & 7 & WCAT1.67sA16mm & 18 & 0.010 & 168 & 7 \\
\hline WCAT1.38sA18mm & 15 & 0.010 & 140 & 7 & WCAT1.67sA18mm & 19 & 0.010 & 178 & 7 \\
\hline
\end{tabular}

Table 5. Mean streak spacing $\bar{\lambda}$, tests conducted in the $16 \mathrm{~m}$ flume.

\begin{tabular}{|c|c|c|c|c|c|c|c|c|c|}
\hline Case Conditions & $\bar{\lambda}(\mathrm{mm})$ & $\begin{array}{c}u_{* \mathrm{c}} \text { or } \\
u_{* \mathrm{wc}} \\
(\mathrm{m} / \mathrm{s})\end{array}$ & $\lambda^{+}$ & $y^{+}$ & Case Conditions & $\bar{\lambda}(\mathrm{mm})$ & $\begin{array}{c}u_{* \mathrm{wc}} \\
(\mathrm{m} / \mathrm{s})\end{array}$ & $\lambda^{+}$ & $y^{+}$ \\
\hline CAA & 17 & 0.0078 & 125 & 6 & WCAAT2sA70mm & 18 & 0.0079 & 135 & 6 \\
\hline WCAAT1.67sA120mm & 18 & 0.0078 & 133 & 6 & WCAAT1.43sA120mm & 18 & 0.0080 & 137 & 6 \\
\hline WCAAT1.25sA100mm & 17 & 0.0085 & 137 & 6 & WCAAT1.11sA86mm & 17 & 0.0085 & 137 & 6 \\
\hline WCAAT1sA70mm & 16 & 0.0082 & 124 & 6 &
\end{tabular}

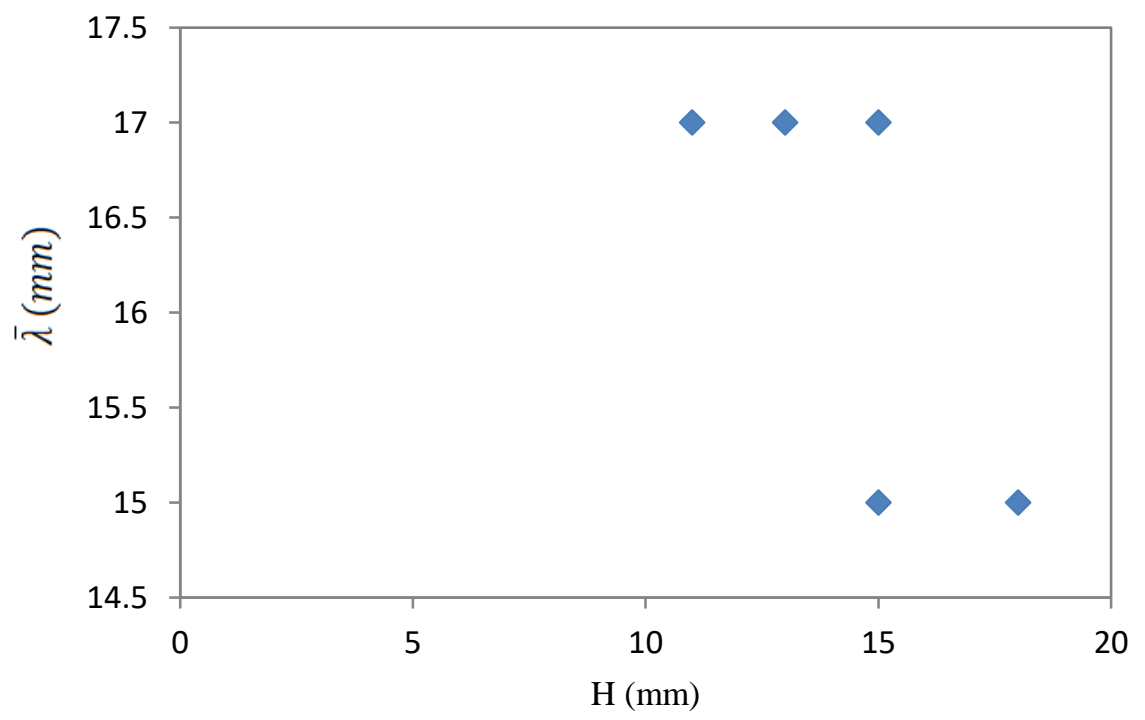

Figure 8. Effect of wave heights on the mean spacing between low-speed streaks: combined wave-current conditions, wave periods $\mathrm{T}=1.38 \mathrm{~s}$. 


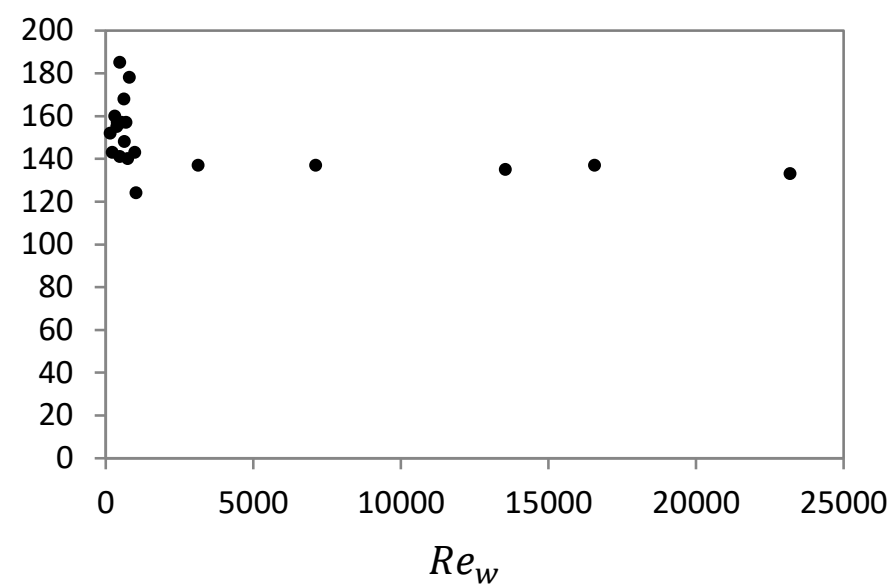

Figure 9. Non-dimensional mean streak spacing for combined wave-current boundary layers.

Flow visualisation shows that streak spacing varies periodically within a wave cycle. This is evidenced by a typical sequence of hydrogen bubble images (Figure 10) and PIV measurements for the low-turbulence flume test (Figure 11), and velocity contours for the $16 \mathrm{~m}$ flume test (Figure 12). For the hydrogen bubble visualisation results, each line of bubbles was created by a separate electrical current 'pulse' (see section 2.3 for details of the instrument); these are also referred to as timelines. The pulsing frequency was kept constant throughout the experiments. The electrical current in the water produced by the pulse was detected by the wave probe and led to the frequent spikes in wave probe signals observed as the zigzag behaviour in Figure 10(c). Low-speed streaks and high-speed regions were detected from the traces of the bubbles. For the PIV results, the field-averaged streamwise velocity is also given in each contour plot to indicate the value of $\tilde{u}$. It should be noted that a phase angle of 0 corresponds to the zero upcrossing point of the wave.

The streak spacing increases when the flow accelerates under a favourable pressure gradient. Figure 10 (b) shows fewer coherent streaks and a more chaotic flow pattern at the phase of maximum near-bed streamwise velocity. Timelines are still 'wavy' and undulated, but the amplitude is less than those observed in Figure 10 (a). This suggests a more uniform distribution of velocities across the flume and will be further discussed later. No intertwined timelines are observed, which can be ascribed to a higher velocity and hence a larger gap between timelines. The PIV measurements [Figure 11 (a)] show that when the streamwise velocity reaches its maximum value, fewer streaks are observed than shown in Figure 11 (b). Results for the $16 \mathrm{~m}$ flume tests show that high-speed regions are wider, leading to a larger streak spacing when the near-bed flow accelerates [Figure 12 (a)].

When the flow decelerates under an adverse pressure gradient, more streaks are observed leading to a decrease of $\lambda$. Figure 10 (a) shows that the first and second time lines of the hydrogen bubbles are very 'wavy'. More streaks are found, as highlighted using green arrows. Many small-scale vortices are observed as detected by the intertwined and kinked timelines (highlighted using orange arrows). The existence of these vortices leads to a decrease of $\lambda$, compared with the turbulent current without waves added. When the distance between the timelines reaches its minimum value during the wave cycle, this indicates that the near-bed $(\mathrm{y}=0.8 \mathrm{~mm})$ streamwise velocity has reached its minimum value. The wave phase at this point is $20^{\circ}$ in advance of the wave trough, which is smaller than the theoretical value of phase advance in classical wave theory (Lamb, 1980). This can be explained by the position of the wire $(\mathrm{y}=0.8 \mathrm{~mm})$, which is near the edge of the wave boundary layer and the finite thickness of the laser light sheet over which the velocity is being averaged. More streaks are observed when the streamwise velocity reaches the minimum value, as seen from the PIV 
measurements for the same test condition [highlighted using ellipses in Figure 11 (b)]. Since the sampling frequency of the PIV measurements is lower than that of the hydrogen bubble visualisation, the actual phase for each PIV image is more uncertain. This leads to the different values of phase angles for the minimum velocity. It should also be noted that the thickness of the PIV laser light sheet can also influence the phase advance value for the PIV measurements because the upper edge of the laser was outside the wave boundary layers. Results from the $16 \mathrm{~m}$ flume tests show the same process of more streaks appearing and $\lambda$ decreasing when the flow decelerates [Figure 12 (e)].

Typical examples of flow visualisation are presented as above, and similar examples were observed during other wave cycles for the same test condition and for other test conditions.

The impact of flow reversal on low-speed streaks can be seen in Figures 12 (c) and (e), with near-bed streamwise velocities of $-0.043(\mathrm{~m} / \mathrm{s})$ and $-0.065(\mathrm{~m} / \mathrm{s})$ respectively. Note that because of the relavely large time steps between consecutive velocity values in the wave cycle, the figures are the first recorded after flow reversal and these correspond to the values of velocity quoted. Assuming that the Taylor hypothesis is also applicable within such a short time between the two images $(d t=0.14 \mathrm{~s})$, any streak would be expected to move backwards for a distance of $6 \mathrm{~mm}$. One well-organised streak, highlighted by the ellipse in Figure 12 (c), is observed to move as expected and to break up into patches of low-speed regions, losing its coherence in the streamwise direction. Analysis of many wave cycles indicates that the extent of this destructive influence coming from flow reversal depends on the strength of the original streaks and the flow velocity. Note that the flow reversal velocity is $-0.065(\mathrm{~m} / \mathrm{s})$ in the present study and those streaks with relatively large widths are not broken down. However, combined wave-current flows with stronger negative velocities may be more effective in breaking up the streaks into spots. This needs further study.

Accompanying the periodic variation of streak spacing, velocity distributions across the flume are changed periodically within a wave cycle. This has been observed from hydrogen bubble visualisation and further confirmed by PIV contours of the transverse velocity gradient $d u / d z$ within a wave cycle [Figure 12 (b), (d) and (f)]. As can be seen from Figure 12 (b), the field of view is more populated with green colours suggesting $d u / d z$ approaches zero when the flow accelerates. This leads to a more uniform distribution across the flume when the flow accelerates. The opposite process is observed when the flow decelerates, with the magnitude of $d u / d z$ increasing and velocity distributions less uniform across the flume. This can be seen from Figures 12 (d) and (f), with more red and blue colours appearing suggesting larger values of $d u / d z$. Since a pair of positive and negative $d u / d z$ indicates where turbulent streaks locate, results are consistent with the streak spacing variation within a wave cycle.

Curve-fitting suggests that the periodic streak spacing within a wave cycle can be represented by a sinusoidal function of time. Figure 13 shows a typical example, and similar results are found in the other flume. The variation of streak spacing is as observed qualitatively in the images shown above. Amplitudes of $\tilde{\lambda}$ are determined from the curve-fitted functions. Considering that only 5 or 10 measurements are obtained per wave cycle, the precise phase for each image is uncertain in this process. This also explains the different values of phase angle corresponding to the minimum velocity (Figure 10). It should also be noted that the PIV measurements in the $\mathrm{x}, \mathrm{y}$ plane cannot get close to the bed. So this cannot be used to measure phase advance. Because of the finite thickness of the light sheet, there is some phase averaging across the wave boundary layer. Therefore, phase angles will not be further discussed here.

A typical example of the distribution of $\lambda$ shows that a Burr distribution is a better description than a lognormal one (Figure 14). Similar distributions are observed in the other facility. This suggests that probabilistic models conform to the same function with and without waves 
added, demonstrating that the fundamental physical process of streak spacing is essentially similar.

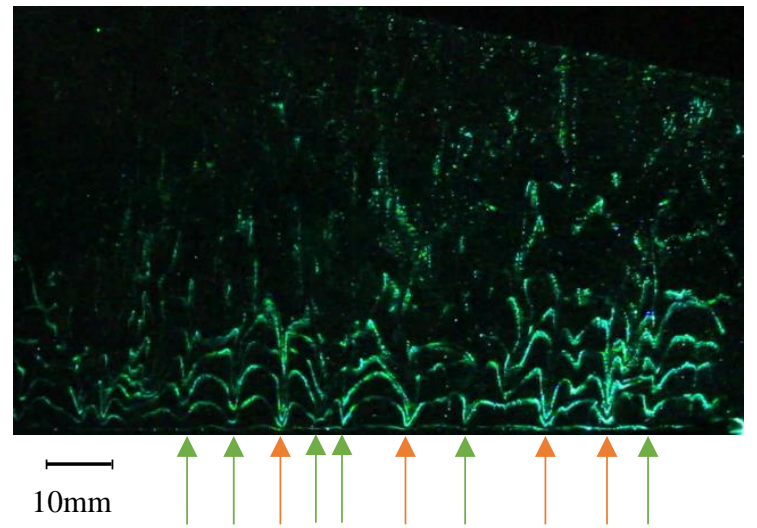

(a) $\mathrm{t}=65.22 \mathrm{~s}$; Phase angle $\psi=249$

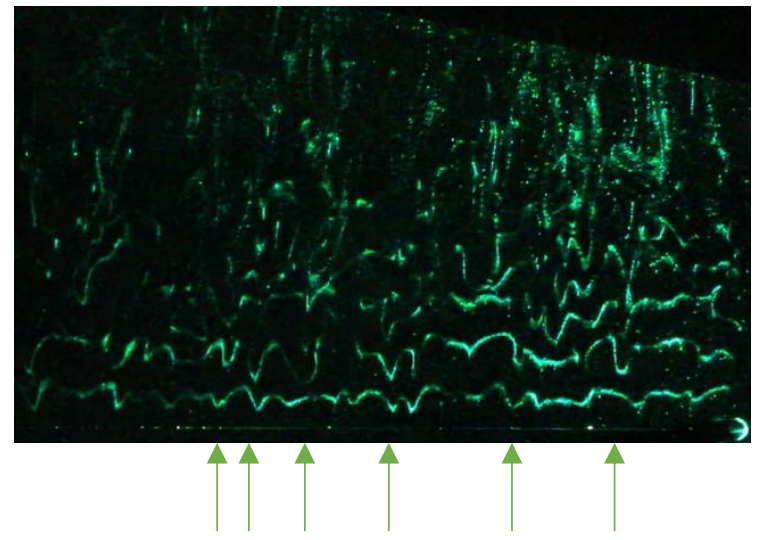

(b) $\mathrm{t}=65.9 \mathrm{~s}$; Phase angle $\psi=67$

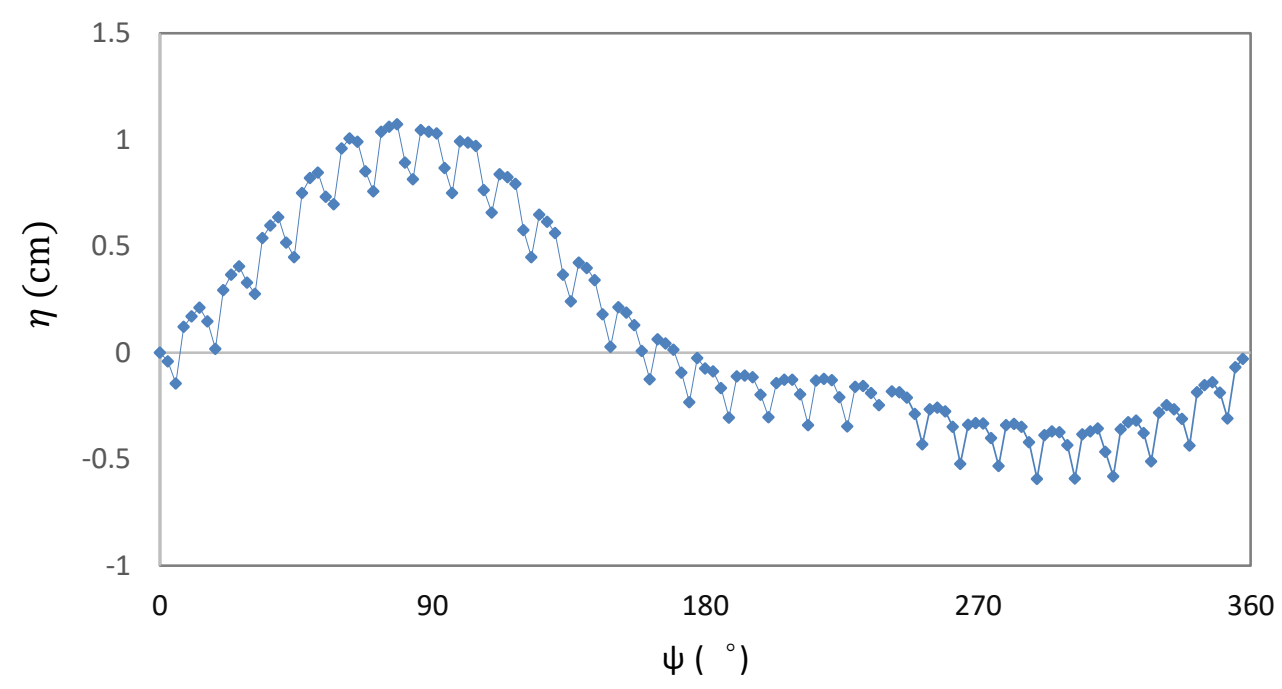

(c)

Figure 10. A sequence of hydrogen bubble visualisations within a wave cycle, combined wave-current flow, low-turbulence flume: wave period $\mathrm{T}=1.38 \mathrm{~s}$, amplitude $\mathrm{A}=18 \mathrm{~mm}$. Wire positioned at $\mathrm{y}=0.8 \mathrm{~mm}$ above the bed, laser light sheet illuminated from the right edge of the image towards left, current flows from bottom of the image upwards: (a) maximum streamwise velocity; (b) minimum streamwise velocity; (c) free surface elevations. 
Field-averaged $\mathrm{U}=\mathbf{0 . 2 0 4}(\mathrm{m} / \mathrm{s})$

Instantaneous streamwise velocities $(\mathrm{m} / \mathrm{s})$

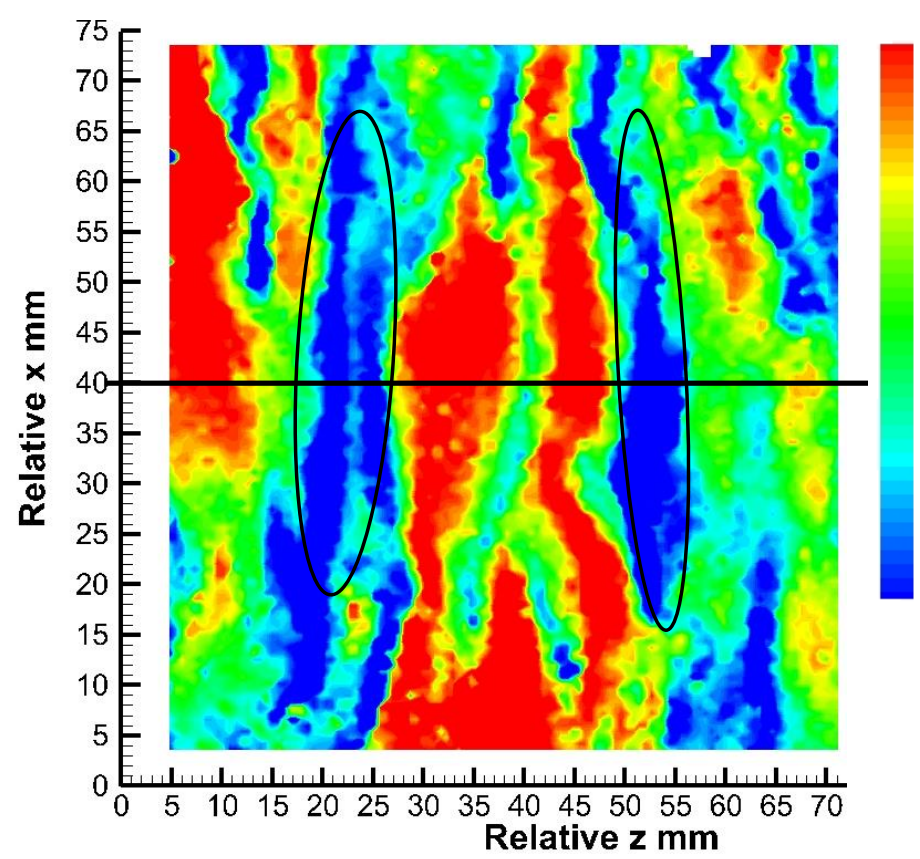

(a) $\mathrm{t}=23.59 \mathrm{~s} ;$ Phase angle $\psi=90$

\subsection{4}

0.228

0.202

0.213

0.205

0.197

0.189

0.182

0.174
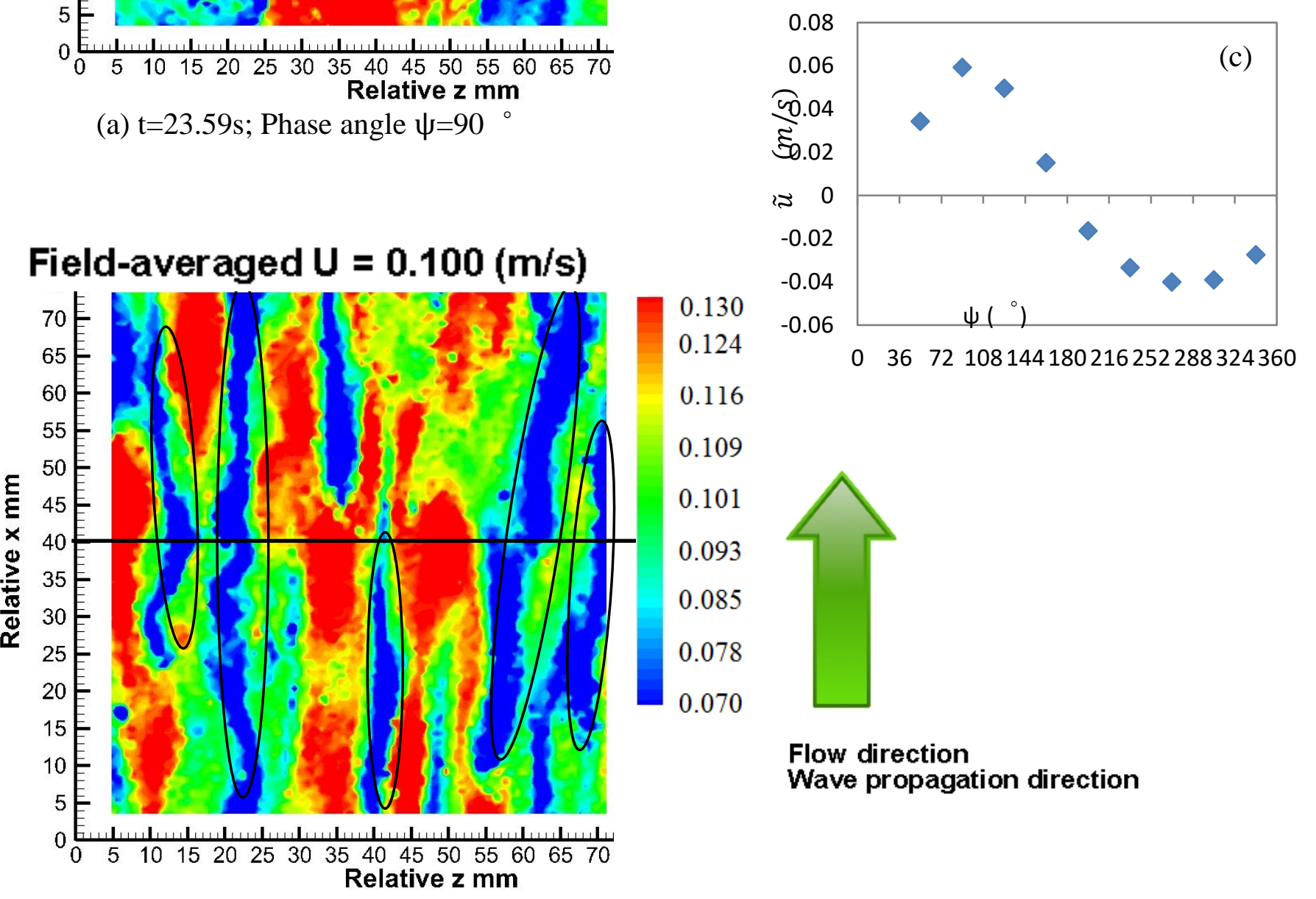

(b) $\mathrm{t}=24.28 \mathrm{~s} ;$ Phase angle $\psi=270^{\circ}$

Figure 11. Evolution of streaks within a wave cycle $(\mathrm{T}=1.38 \mathrm{~s}, \mathrm{~A}=18 \mathrm{~mm}), \mathrm{y}=0.8 \mathrm{~mm}$ above the bed, current flows from bottom of the image upwards: (a) maximum streamwise velocity, PIV measurements; (b) minimum streamwise velocity, PIV measurements; (c) wave-induced periodic streamwise velocities, PIV measurements. 
Field-averaged $\mathrm{U}=\mathbf{0 . 3 3 8}(\mathrm{m} / \mathrm{s})$

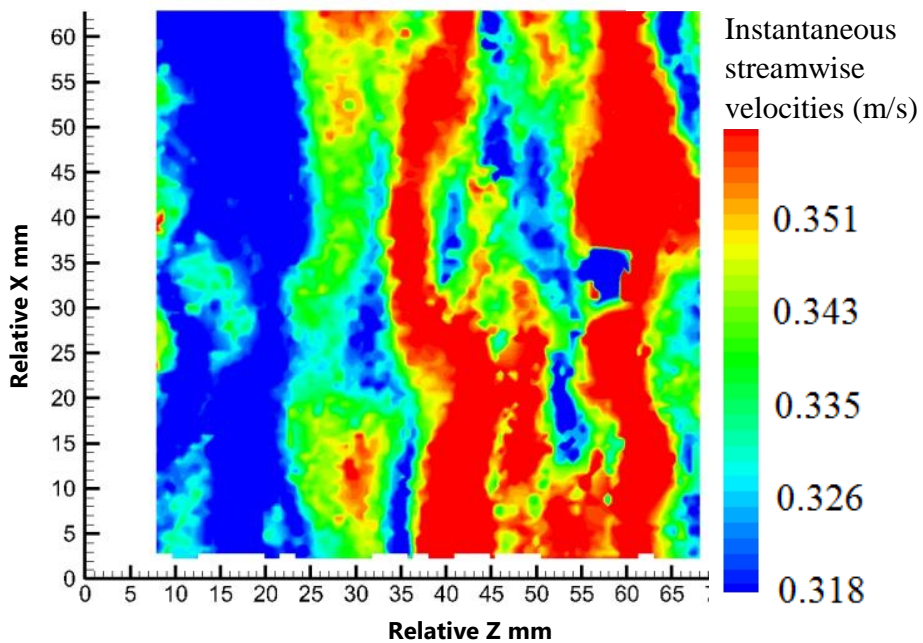

(a) $\psi=93$

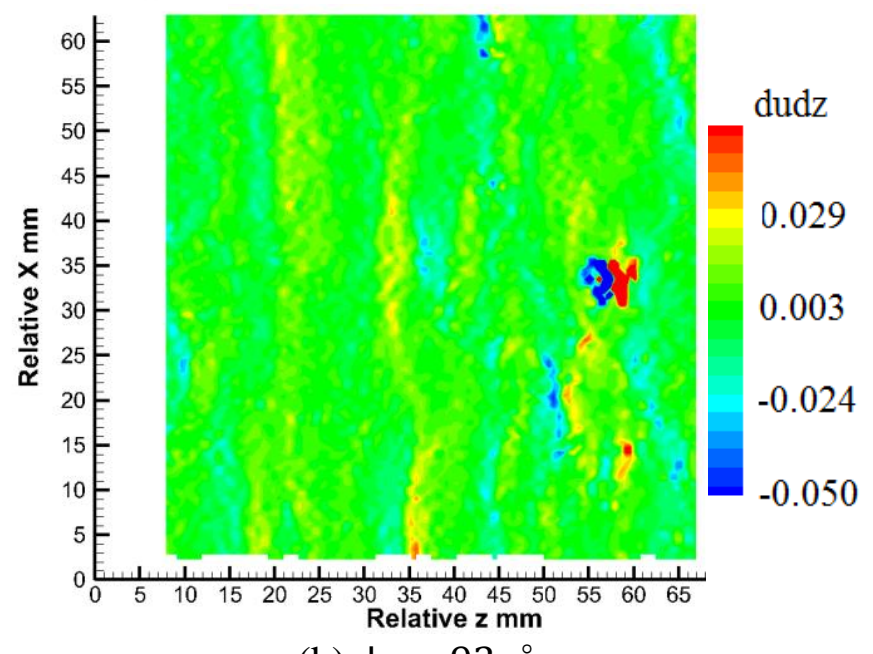

(b) $\psi=93$

Field-averaged $\mathrm{U}=\mathbf{-} 0.043(\mathrm{~m} / \mathrm{s})$

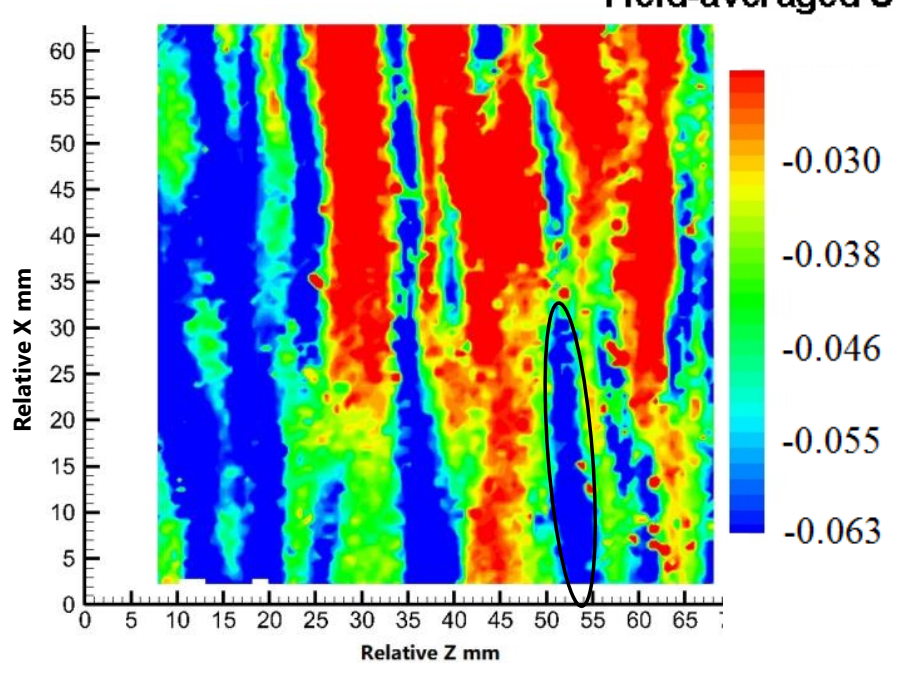

(c) $\psi=238$

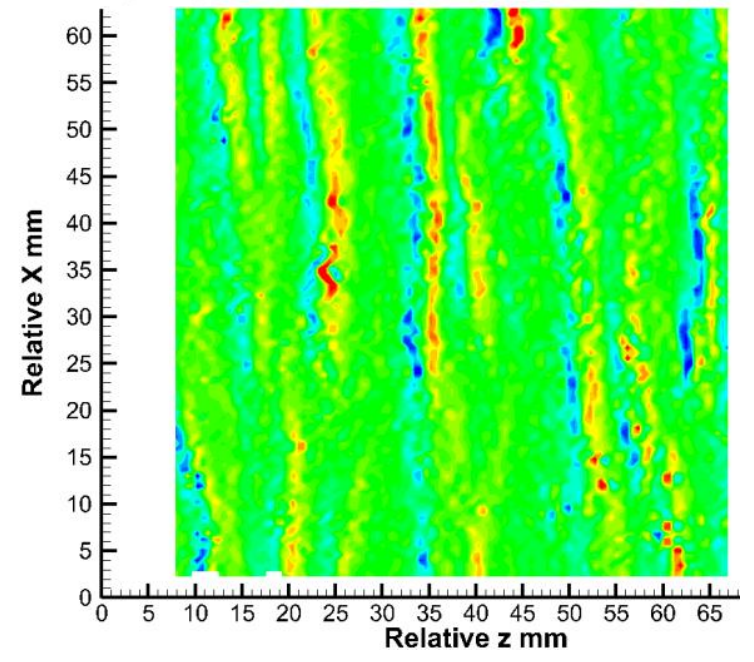

(d) $\psi=243$

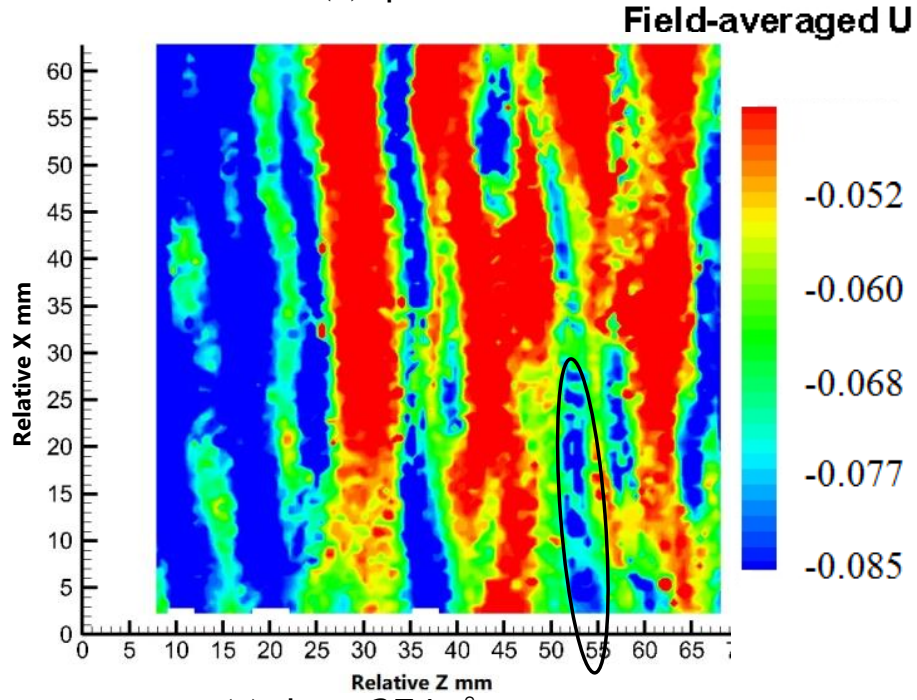

(e) $\psi=274$

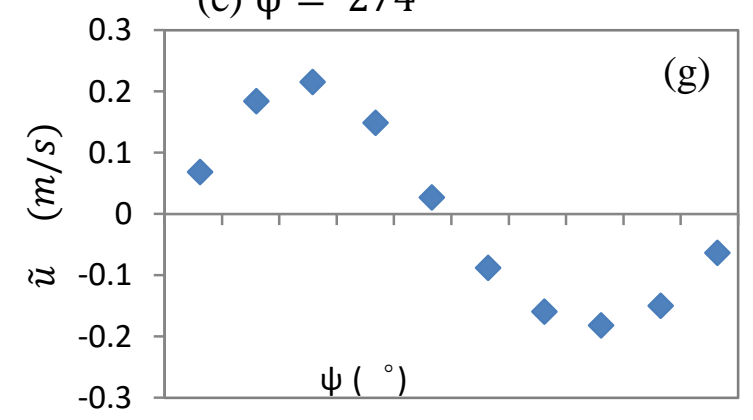

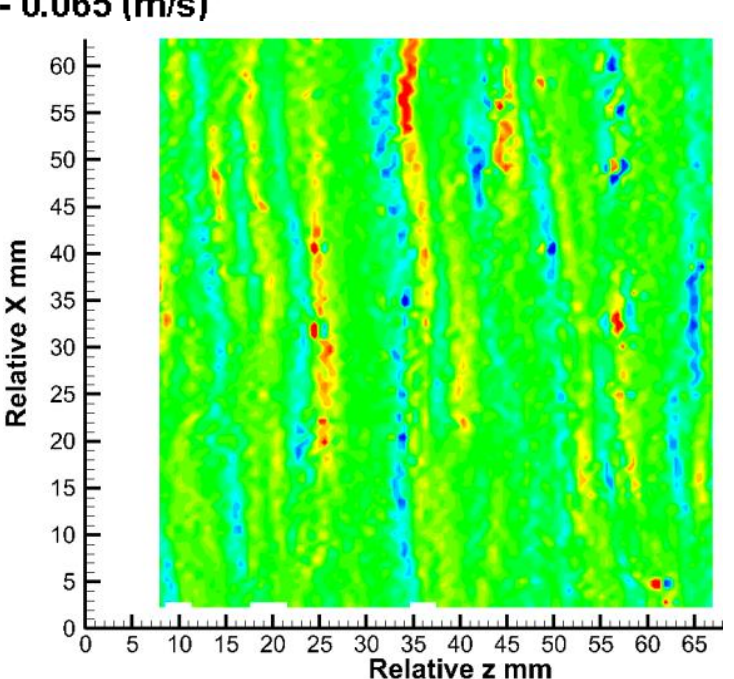

(f) $\psi=279$

Figure 12. Evolution of streaks within a wave cycle $(\mathrm{T}=1.43 \mathrm{~s}, \mathrm{~A}=120 \mathrm{~mm})$, PIV measurements at $\mathrm{y}=$ $0.8 \mathrm{~mm}$, water depth of $400 \mathrm{~mm}, 16 \mathrm{~m}$ flume: (a), (c) aflel (e) showing contours of streamwise velocities $u$ $(\mathrm{m} / \mathrm{s}) ;(\mathrm{b}),(\mathrm{d})$ and (f) showing velocity gradient $d u / d z\left(10^{3} \times \mathrm{s}^{-1}\right) ;(\mathrm{g})$ presenting the periodic streamwise velocities. 

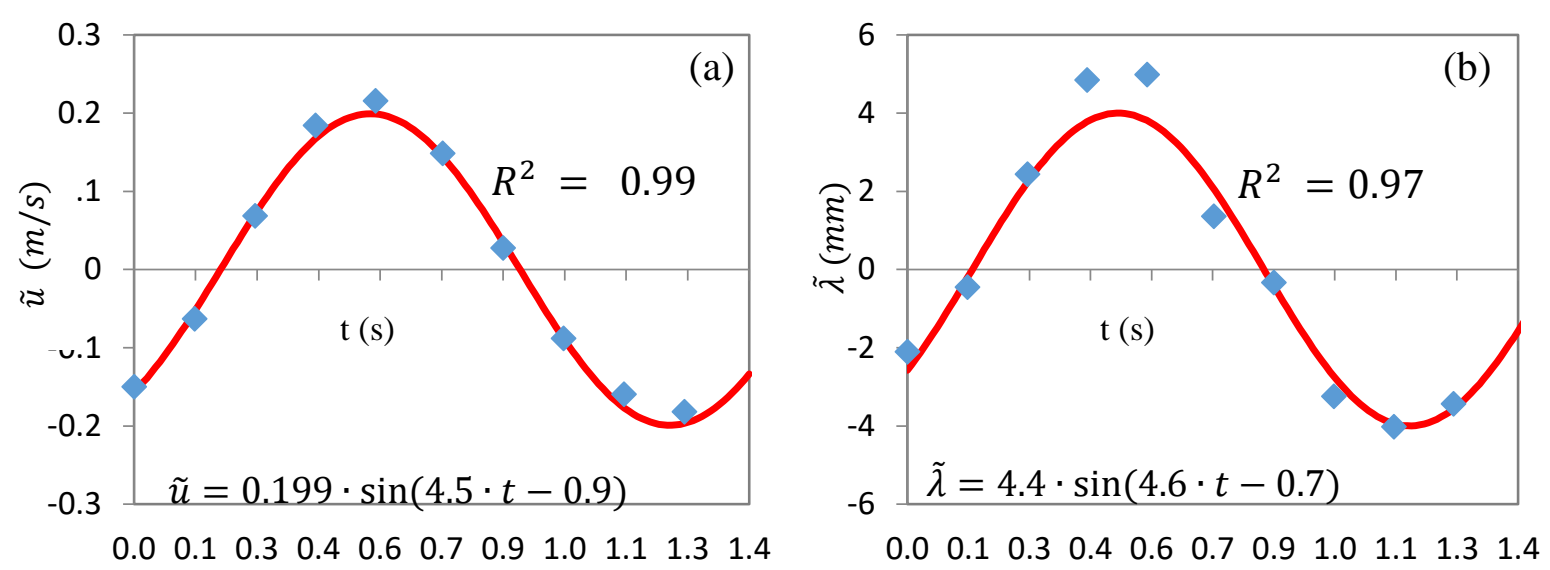

Figure 13. Phase variations for the combined flow in the $16 \mathrm{~m}$ flume: $\mathrm{T}=1.43 \mathrm{~s}, \mathrm{~A}=120 \mathrm{~mm}$, obtained at $\mathrm{y}=0.8 \mathrm{~mm}$, (a) wave-induced streamwise velocity $\tilde{u}(\mathrm{~m} / \mathrm{s})$; (b) periodic streak spacing $\tilde{\lambda}(\mathrm{mm})$. Dots represent experimental results, and lines show a best fit curve-fitting using sinusoidal functions of time.

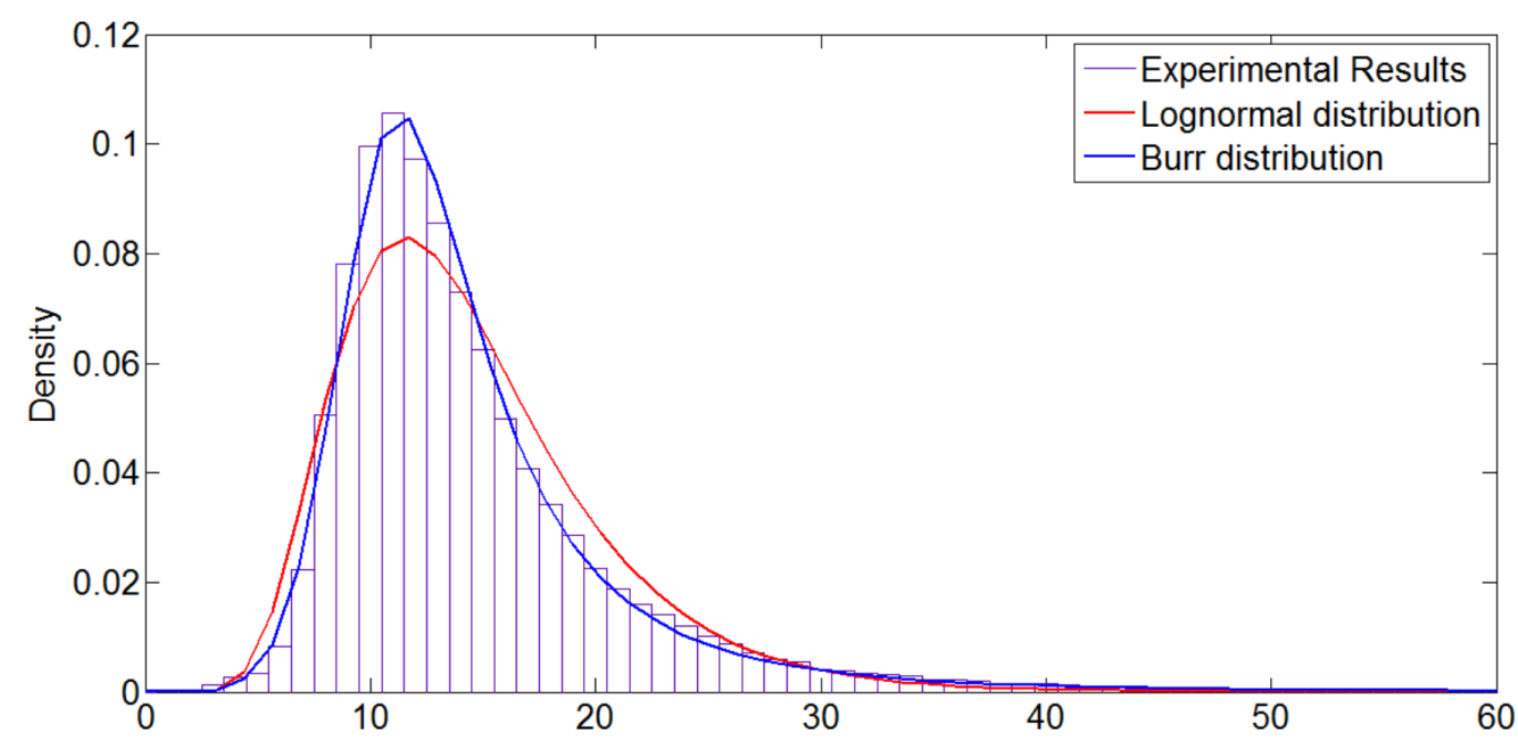

Figure 14. Probability density functions of streak spacing at $y=0.8 \mathrm{~mm}$, low-turbulence flume, combined wave-current flow: wave period $\mathrm{T}=1.38 \mathrm{~s}$, wave amplitude $\mathrm{A}=20 \mathrm{~mm}$.

\subsubsection{Coherent structures: ejections and sweeps}

The kinematics of turbulent vortices are well described by the local ensemble-averaged velocity. A typical example is given in Figure 15, showing contours of vorticity magnitude overlaid by velocity vectors within a wave cycle. Note that velocity vectors plotted here are the instantaneous turbulent fluctuations. No additional moving reference frame is needed. Shear layers detected by the ejections residing beneath them ('S1' and 'S2') move downstream approximately at the local velocity [Figures 15 (a) and (b)]. Vortices denoted 'A', 'B', 'C', 'D' and 'E' show the same behaviour [Figures 15 (c) to (e)]. The angle of orientation denoted as $\alpha$ increases from Figure 15 (c) to (d). This is explained by the additional spanwise rotation induced by wave-induced flow reversal below $y=17 \mathrm{~mm}$ observed from the ensemble-averaged velocity profiles. After flow reversal [Figures 15 (d) to (e)], the vortices are lifted up into the outer region and move forwards under the action of potential flow. 
When the flow decelerates, ejections are stronger and the size of vortices increases. This is seen from Figures 15 (a) to (d). More violent ejections are observed, as evidenced by increasing length scales of arrows around the vortex core region denoted as ' $E$ '. The diameter of these vortices, as highlighted using red ellipses and denoted as 'A', 'B', and 'C', evolves from $4 \mathrm{~mm}$ to $6 \mathrm{~mm}$. Vortices are more circular.

Vortices are split into smaller ones when the flow accelerates. This is illustrated from Figures 15 (d) to (e). The vortex labelled ' $\mathrm{D}$ ' [Figure 15 (d)] is observed to be split into two smaller vortices, denoted as 'D1' and 'D2', in Figure 15 (e).

The process of vortices increasing in scale when the flow decelerates and being split into several smaller vortices when the flow accelerates has some scientific implications. According to the energy cascade theory of turbulent boundary layers (Kolmogorov, 1941), vortices of larger scale normally generate turbulent kinetic energy and pass energy to those of smaller scale. Smaller vortices then consume turbulent kinetic energy, mainly by viscosity. The observed phenomenon reveals the underlying physical process of turbulence generation when flow decelerates under an adverse pressure gradient, and suppression when it accelerates under a favourable pressure gradient (Hino et al., 1983).

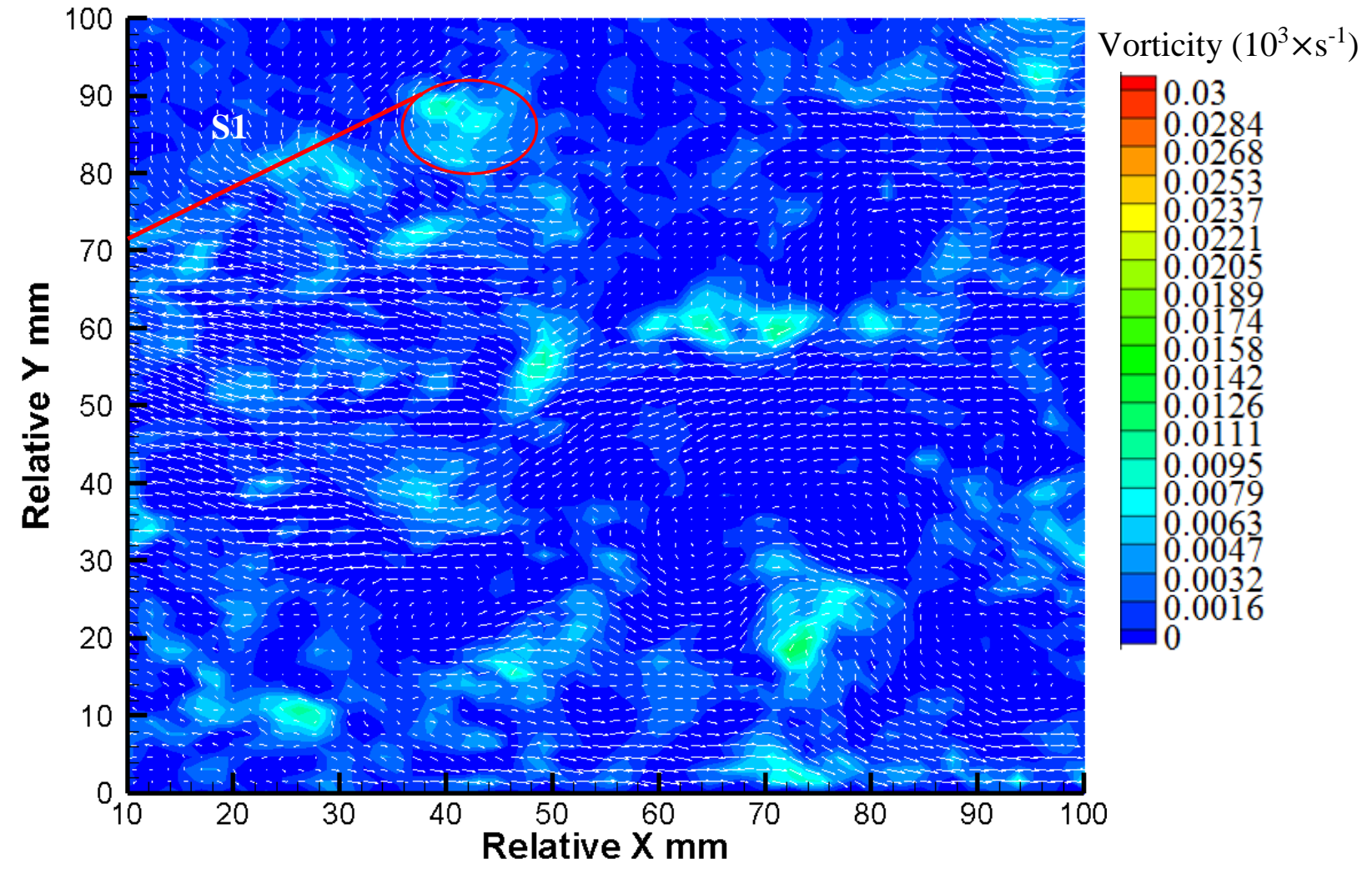

(a) $t=26.7 \mathrm{~s} ;$ phase angle $=72^{\circ}$ 


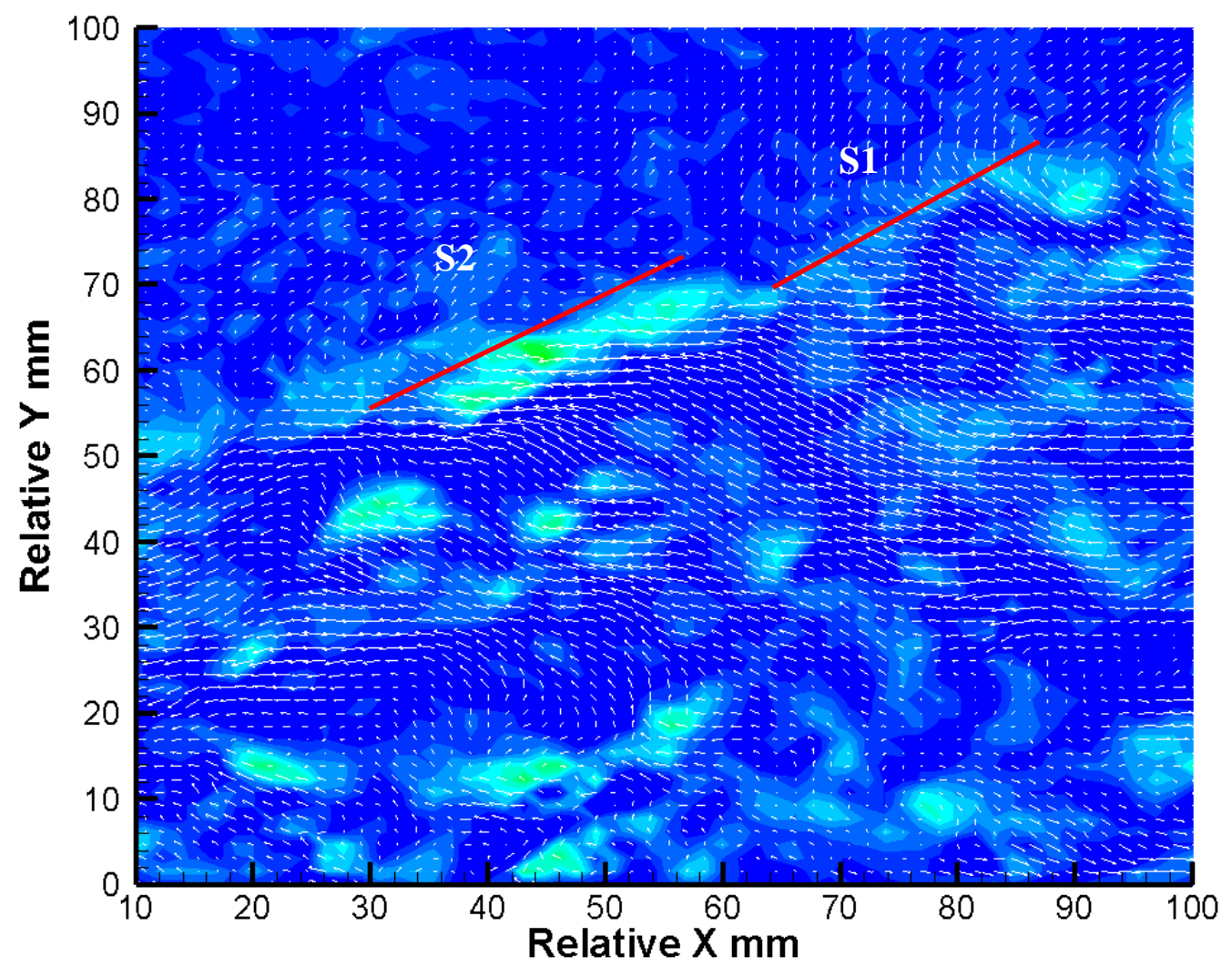

(b) $t=26.84 \mathrm{~s}$; phase angle $=108^{\circ}$

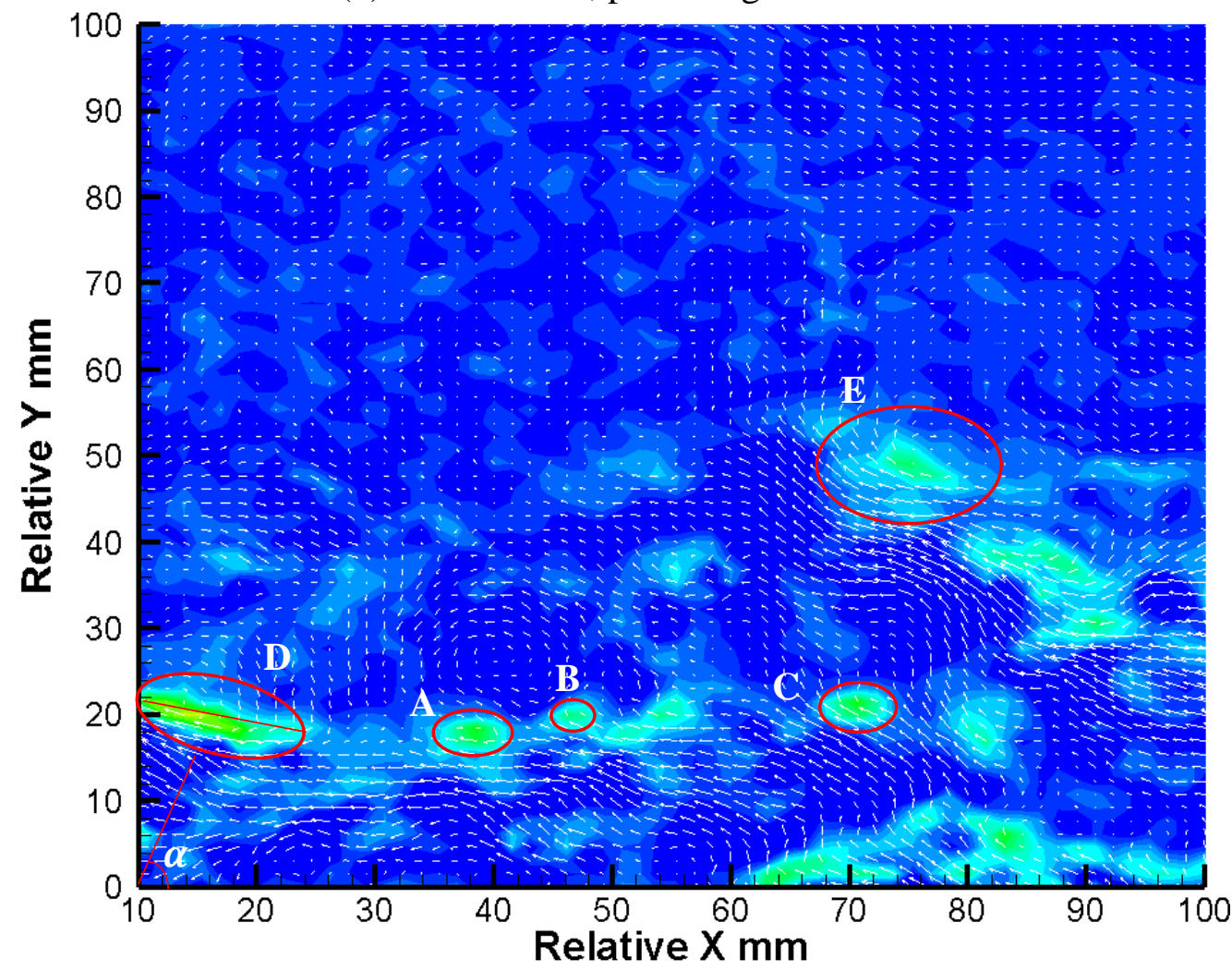

(c) $t=27.28 \mathrm{~s} ;$ phase angle $=216^{\circ}$ 


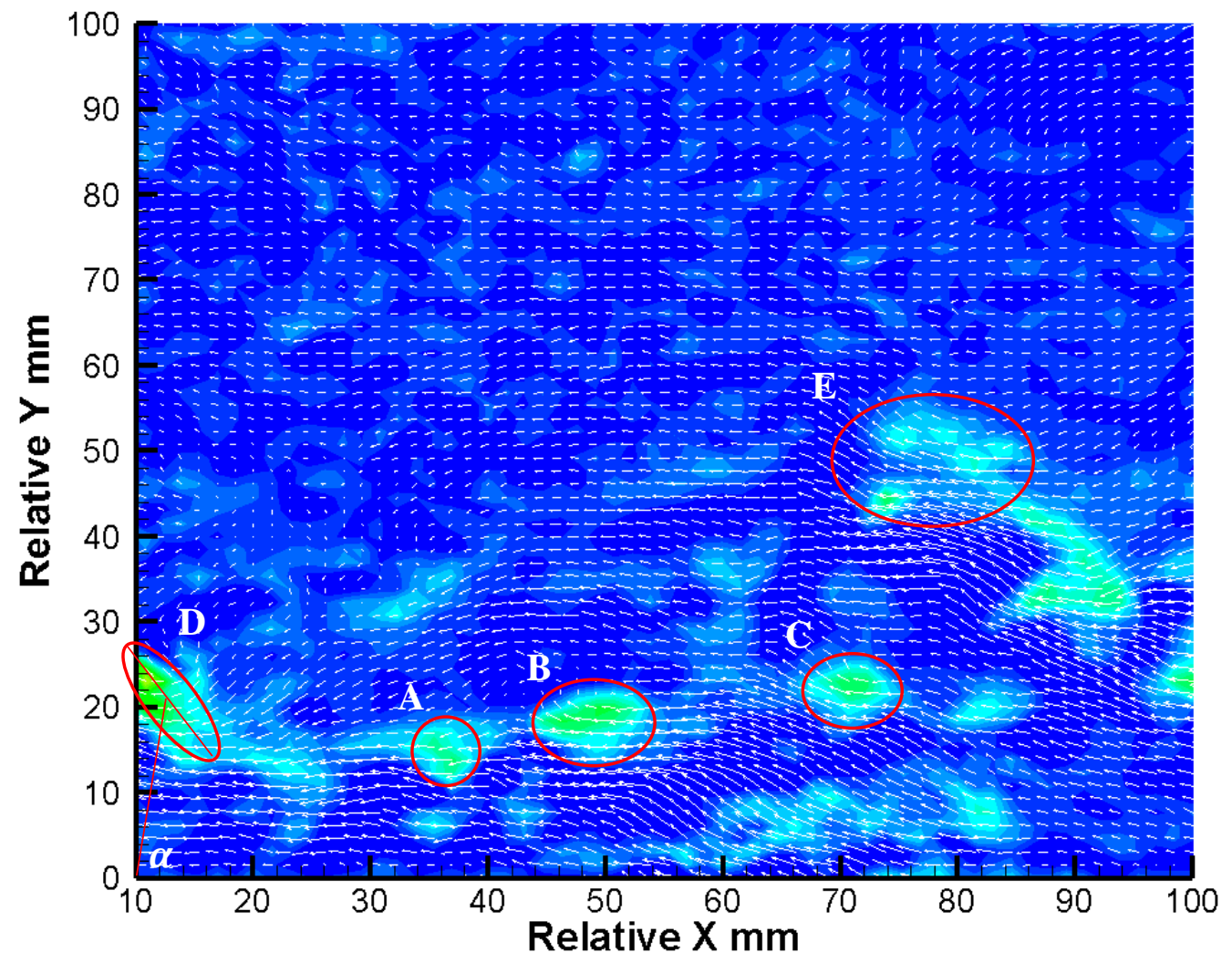

(d) $t=27.56 \mathrm{~s} ;$ phase angle $=288^{\circ}$

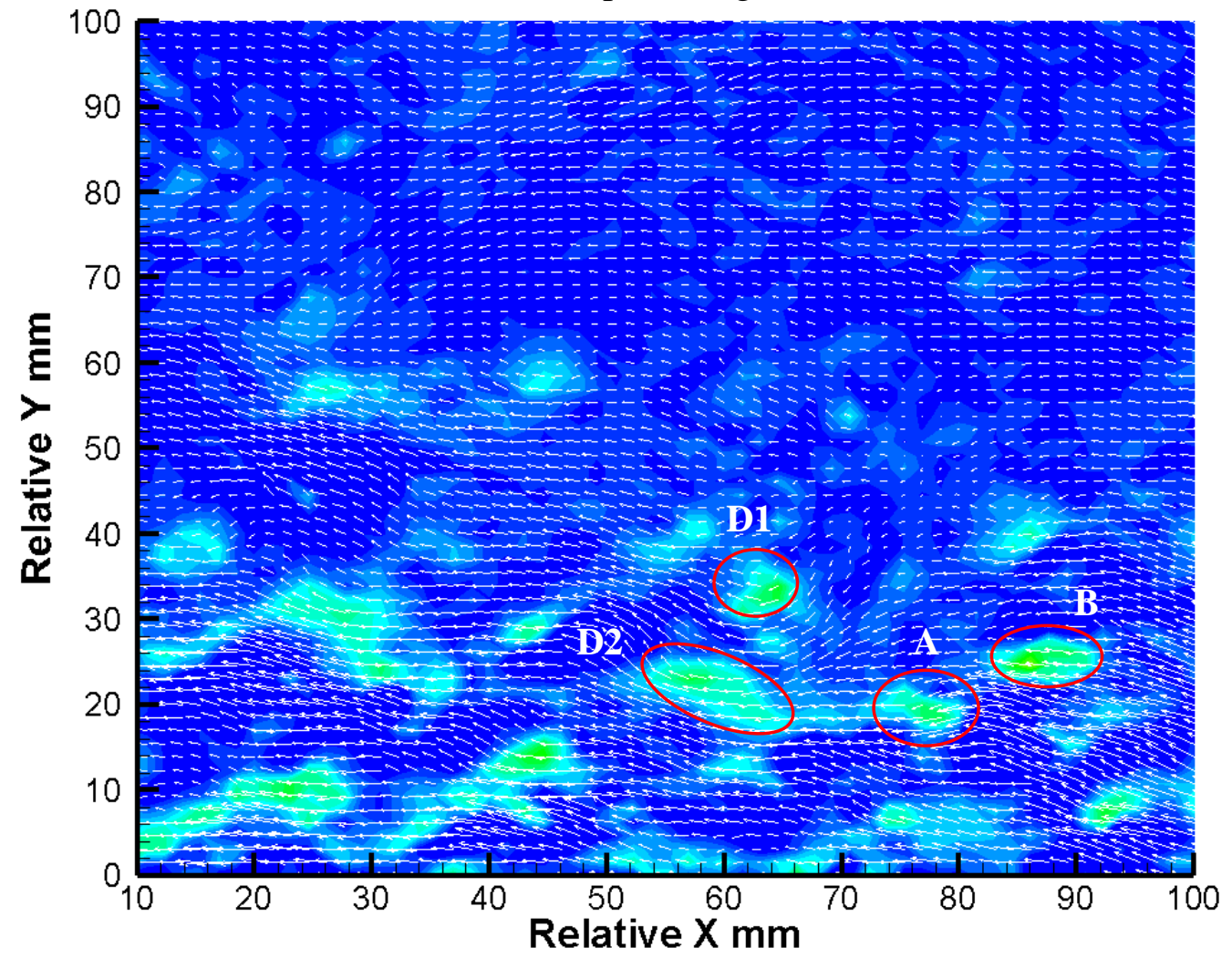

(e) $t=28 \mathrm{~s}$; phase angle $=36^{\circ}$

Figure 15 . Vorticity $\left(10^{3} \times \mathrm{s}^{-1}\right)$ within a wave cycle in the combined wave-current boundary layer: wave period $T=1.43 \mathrm{~s}$, amplitude $A=120 \mathrm{~mm}, 16 \mathrm{~m}$ flume. 
Waves with larger amplitudes lead to larger shear stress induced by ejections. The effect of wave amplitude is illustrated in Figure 16, showing Reynolds shear stress contributed from the four quadrant events (sweeps, ejections and interactions). Note that all magnitudes of Reynolds shear stress are divided by the density of water. Graphs only show the results within the boundary layers, as evidenced by the Reynolds shear stress and mean velocity profiles. Results show that the shear stress induced by ejections (Q2 events) is higher with waves of increasing amplitude than in a turbulent current without waves. Results for the other three quadrant events remain similar to the turbulent current flow when waves are not present. Figure 17 shows a typical example of the influence of waves on the shear stress, after normalised by $u_{*}{ }^{2}$. The results show that the contribution from ejections (Q2) is not altered by the waves but that the contribution from sweeps $(\mathrm{Q} 4)$ is reduced. The different roles of Q2 and Q4, as observed from Figures 16 and 17, can be explained by the large increase of shear velocities when waves are superimposed. However, results from both sets of experiments suggest that the ratio of Q2/Q4 is enhanced by the waves added.
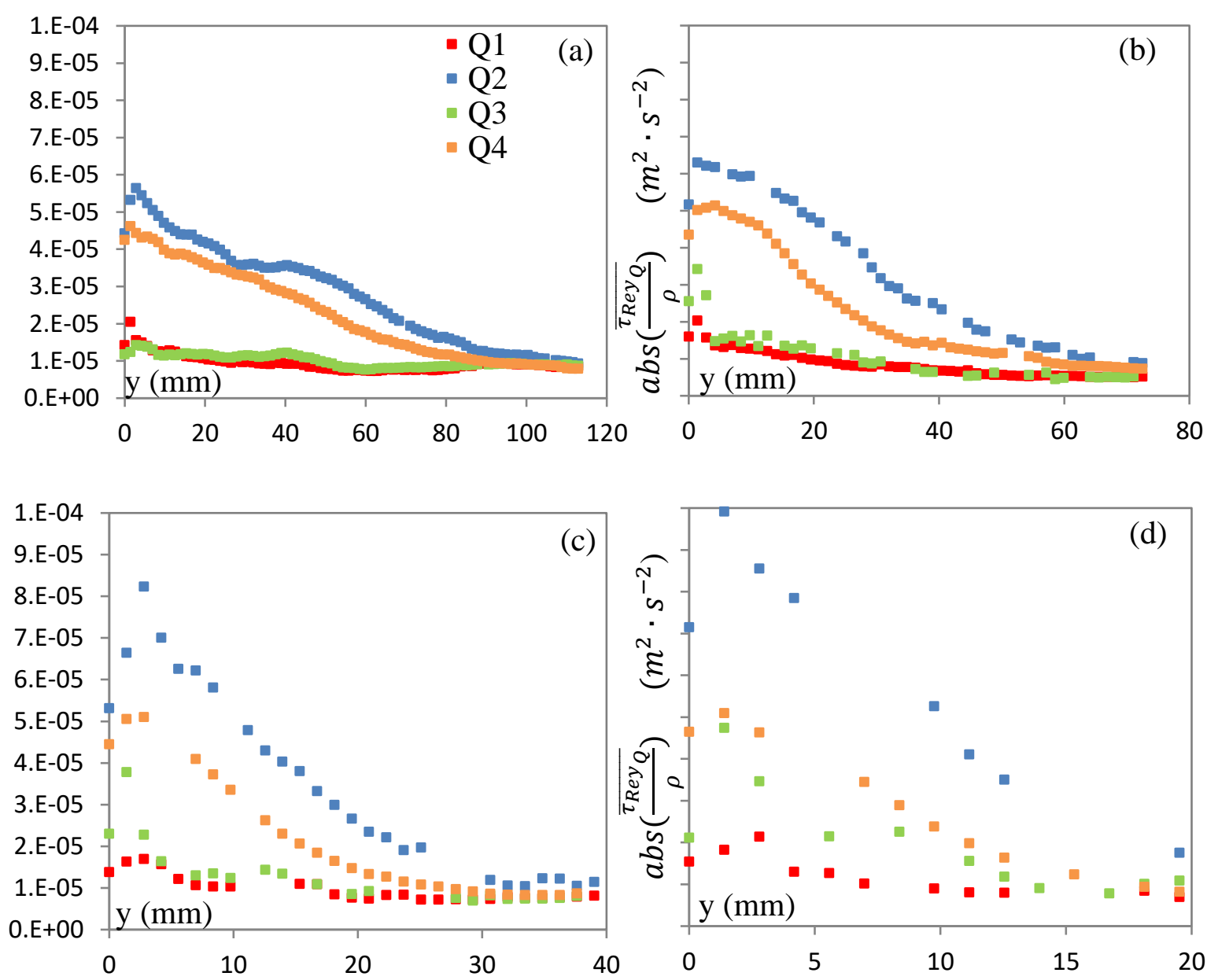

Figure 16. Reynolds shear stress induced by four quadrant events, unidirectional turbulent current with and without waves added, low-turbulence flume: (a) CA; (b) WCAT1.25sA14mm; (c) WCAT1.25sA16mm; (d) WCAT1.25sA20mm. 

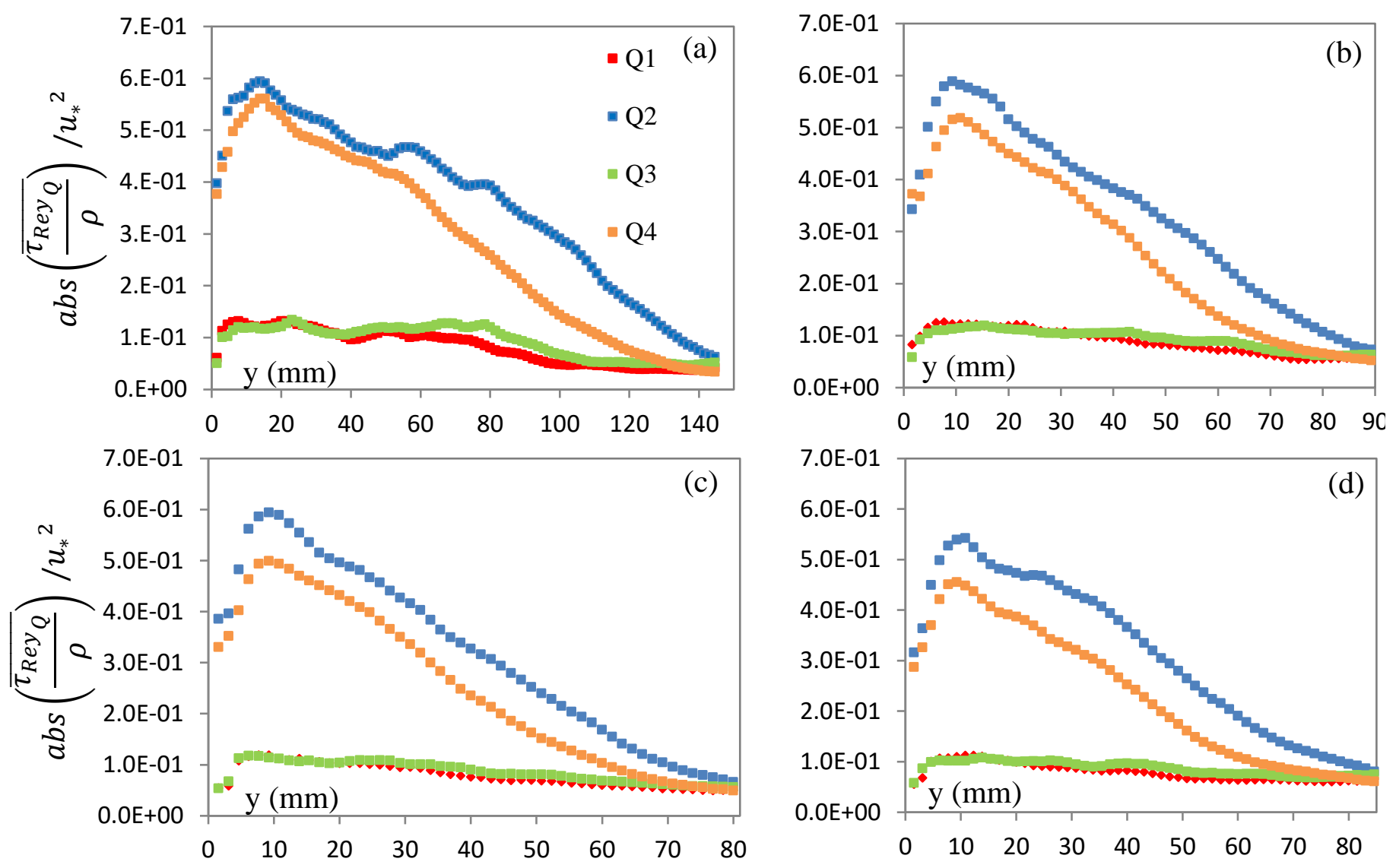

Figure 17. Reynolds shear stress induced by four quadrant events, normalised by squares of shear velocities $\left(u_{* c}^{2}\right.$ or ${u_{* w c}}^{2})$, waves propagate with the turbulent current, 16m flume: (a) CAA; (b) WCAAT1.11sA86mm; (c) WCAAT1.25sA100mm; (d) WCAAT1.43sA120mm.

\section{Discussions}

The amplitude of changes in periodic streak spacing $\tilde{\lambda}_{\text {magnitude }}$ increases with an increasing streamwise velocity magnitude $\widetilde{u}_{\text {magnitude }}$. Further intercomparisons between runs 'WCAT1.25sA20mm', 'WCAT1.38sA20mm', and 'WCAT1.67sA18mm' suggest that longer wave periods can also lead to larger changes. In physical terms it is likely that fluctuations in $\tilde{\lambda}_{\text {magnitude }}$ are related to the orbital excursion induced by waves. In order to investigate this quantitatively, Figure 18 shows the variation of $\tilde{\lambda}_{\text {magnitude }}$ with orbital excursion amplitude $\tilde{a}_{\text {magnitude }}$ for tests in the two flumes. A best-fit (red) line indicates that they are reflected by a linear function, $\mathrm{R}^{2}>0.9$ indicating a good fit. This implies that the forward and backward movement of the boundary layer induced by the waves can change the structure of turbulence. The results presented above have implications for the understanding of turbulent boundary layers. Scandura et al. (2016) found that the low-speed streak spacing increases at $t=\pi / 8$. Low-speed streaks in pure oscillatory flows break down into small segments before the velocity reaches its maximum value. Van der A et al (2018) found a more uniform distribution of the velocity fluctuations, after the breaking of low-speed streaks. The present experiments suggest that the spacing increases (decreases) when the combined wave-current flow accelerates (decelerates). Previous studies in a unidirectional turbulent current suggest that a larger streak spacing can lead to a lower bursting frequency (Johnson, 1998). The finding from the present study indicates that bursting frequencies may decrease when the flow accelerates and increase when the flow decelerates. Based on the conceptual model of 
hairpin vortices, these results also imply that legs of vortices can move further apart when the flow accelerates and more closely together when the flow decelerates.

When waves with shorter periods and larger amplitudes are added, a small but consistent decrease of $\bar{\lambda}$ is observed. This finding is also relevant to the reduction of boundary layer thickness, because the size of turbulent vortices is a characteristic length scale of the boundary layer. However, it should be emphasised that the change in $\bar{\lambda}$ is not of the same magnitude as the decrease of boundary layer thickness. The different changes in $\bar{\lambda}$ and $u_{*}$ induced by waves indicate a new value of $\lambda^{+}$for combined wave-current boundary layers as established from the present study.

The observation above has implications for sediment transport. The streak spacing represents the typical length scale of parting lineation. Therefore, results from the present investigation indicate that the spacing between sediment streaks may decrease where waves and currents coexist, especially when shorter and larger waves appear. The newly obtained knowledge reveals the underlying physical process of streaky patterns in coastal areas and hence provides a way to interprete field data. The knowledge of streak spacing gained from the present investigation may also drive future developments of more advanced sediment transport models. Since most models at present still assume that the bed is uniform, future models may describe the bedform configurations and differentiate areas which are populated by the streaks. Previous studies of sediment transport have found that suspended sediment is closely related to ejections (Lelouvetel et al., 2009). The present results suggest that the extra ejections induced by waves add to diffusion and are a mechanism to keep more sediment in suspension.

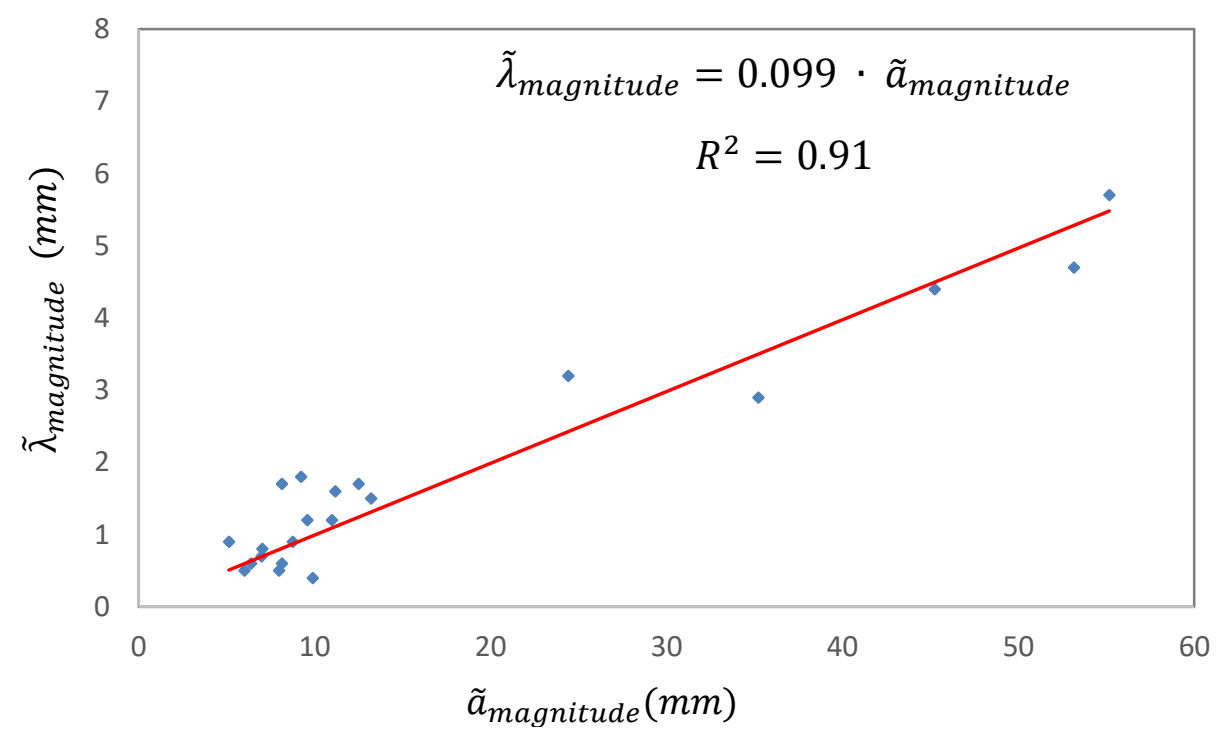

Figure 18. Magnitude of periodic streak spacing vs orbital excursion amplitude.

The streak spacing decreases (increases) when the flow decelerates (accelerates), together with stronger (weaker) ejections. This provides a possible mechanism for turbulence suppression when flow accelerates under a favourable pressure gradient and enhancement when decelerates under an adverse pressure gradient (Hino et al., 1983). By analogy to the self-sustained process of turbulent boundary layers proposed [Kim et al. (1971)], the lifted low-speed streaks interact with the instantaneous velocity profile and cause inflection points. Such inflexion points are indicative of flow instability and lead to ejections thereafter, together with sweeps according to the continuity law. The whole process constitutes a bursting event [Kim et al. (1971)] which is the basic mechanism of turbulence generation. A 
relationship between the streamwise velocity and streak spacing is established, indicating the way of disturbing turbulent boundary layers by wave-induced orbital displacements.

The presence of more streaks when flow decelerates provides an additional mechanism for initiation of sediment movement when waves are added. Previous studies of turbulent boundary layers have shown that streamwise vortices are closely related to high bed friction (Kravchenko et al., 1993), and that pairs of counter-rotating streamwise vortices are located at each side of a streak (Stretch, 1990; Schoppa and Hussain, 2002). This suggests that the bed shear stress is increased when streamwise vortices appear; this, together with a vertical pressure gradient, can initiate movement of sediment particles more easily.

The spanwise distribution of streamwise velocities $u$ across the flume changes periodically within a wave cycle. When $u$ increases (decreases), the distribution is more (less) uniform. This implies that sediment particles on the seabed may agglomerate when the flow accelerates.

Within a wave cycle, the scale of spanwise vortices increases (decreases) when the flow decelerates (accelerates). This suggests that turbulent diffusion may be stronger when the flow decelerates. Sediment particles are entrained by stronger vortices, similar to the boils in open-channel flows.

\section{Conclusions}

\subsection{General conclusions}

This study provides new insight to the structure of turbulence in the bottom wave-current boundary layer. The main conclusions obtained are as follows:

1. Low-speed streaks observed in the unidirectional current boundary layer are also present in combined wave-current boundary layers. A decrease of mean streak spacing of less than $3 \mathrm{~mm}(13 \%)$ is observed when waves with larger amplitudes and higher frequencies are added. The non-dimensional mean streak spacing is $\lambda^{+}=140$, as established from the present study, and is different from that of the unidirectional turbulent current $\left(\lambda^{+}=120\right)$.

2. Within a wave cycle, the spacing between turbulent streaks varies with the passing of wave crest and trough, increasing when the flow accelerates and decreasing when the flow decelerates. The amplitude of the change in periodic streak spacing is linearly related to the wave-induced orbital displacements in the streamwise direction.

3. Velocity gradients across the flume change periodically within one wave cycle. When the flow accelerates (decelerates), streamwise velocities distribute more (less) evenly across the flume.

4. Flow reversal is seen to cause low-speed streaks to break up into blocks of low-speed fluid, losing their coherence in the streamwise direction.

5. For all tests in the present study (current-alone and wave-current conditions), the probability density functions of streak spacing are essentially very similar with and without waves added to the current. Both are better represented by a Burr distribution than by the lognormal distribution found by previous authors for a unidirectional turbulent current.

6. Quadrant analysis of Reynolds shear stress reveals that the maximum Reynolds shear stress induced by ejections is increased when waves are superimposed on the turbulent current. The change scales with wave frequencies and amplitudes. Contributions from the other three quadrant events remain similar to those for the turbulent current without waves.

The findings also provide insight to changes in sediment transport under combined waves and currents. Nakagawa and Nezu (1981) showed that the probability density function for streak spacing in a unidirectional current is the same as that for the bursting frequency. Thus, the PDF of streak spacing found here can be used to derive a new probabilistic model for 
sediment resuspension. And the periodic change of velocity distribution across the flume within a wave cycle indicates a possible movement path for sediment.

\subsection{Remark on rough boundaries}

It should be noted that the experiments were conducted over smooth boundaries. A few studies have been carried out to investigate the bursting process in unidirectional currents over both smooth and rough boundaries (Grass et al., 1991, 1993). Their work demonstrated that turbulent structures over smooth and rough boundaries are essentially the same. The more recent work of Carstensen et al. (2012) has revealed the existence of coherent structures in combined wave-current flows and showed that turbulent spots are detected over both smooth and rough boundaries. It is therefore hypothesised that results from the present investigation may be generalised to flow fields over rough boundaries in wave-current boundary layers. However, this hypothesis needs further work since there is as yet no study on the bursting process in combined wave-current boundary layers over a rough boundary.

\section{List of Symbols}

\section{Symbol Description}

$f \quad$ Sampling frequency

$h \quad$ Water depth

$n \quad$ Index number of time sequence of velocity measurements

$d t \quad$ Time between two consecutive measurements

$t \quad$ Time

$u \quad$ Instantaneous streamwise velocity

$v \quad$ Instantaneous vertical velocity

$w \quad$ Instantaneous spanwise velocity

$x \quad$ Horizontal displacement

$y \quad$ Vertical displacement above the bed

$z \quad$ Transverse displacement

$\kappa \quad$ Von Kármán constant

$\rho \quad$ Density of water

$v \quad$ Kinematic viscosity of water

$\Delta \quad$ Grid size in the spanwise direction

$x_{i_{1}} \quad$ Streamwise displacement relative to the left edge of the PIV image

$y^{+} \quad$ Non-dimensional vertical displacement above the bed

$\overline{\tau_{R e y}} \quad$ Time-averaged Reynolds shear stress

$\overline{\tau_{R e y_{Q}}} \quad$ Magnitude of time-averaged Reynolds shear stress contributed from each quadrant event

$\bar{U} \quad$ Mean velocity

$u(z)^{\prime \prime} \quad$ Velocity fluctuation in the spanwise direction

I.S.S Instantaneous integral spatial scale

$z_{0} \quad$ First zero-crossing point of the auto-correlation coefficient distributions

$\lambda \quad$ Instantaneous value of streak spacing

$\bar{\lambda} \quad$ Time-averaged mean streak spacing

$\sigma_{\lambda} \quad$ Standard deviation of streak spacing

$\psi_{\lambda} \quad$ Coefficient of variation of streak spacing

$S_{\lambda} \quad$ Skewness of streak spacing

$F_{\lambda} \quad$ Flatness of streak spacing

$P \quad$ Probability density function 


$\begin{array}{ll}\text { Symbol } & \text { Description } \\ \xi & \text { Shape parameter of Burr probability density function } \\ \Omega & \text { Shape parameter of Burr probability density function } \\ Y & \text { Scale parameter of Burr probability density function } \\ \lambda^{+} & \text {Non-dimensional mean spanwise spacing } \\ u_{*} & \text { Shear velocity, either in a current-alone condition }\left(u_{* c}\right) \text { or a combined } \\ R^{2} & \text { wave-current flow }\left(u_{* w c}\right) \text {. } \\ \tilde{\lambda}_{\text {magnitude }} & \text { Coefficient of determination } \\ A & \text { Amplitude of changes in periodic streak spacing } \\ H & \text { Wave amplitude } \\ T & \text { Wave height } \\ T_{B} & \text { Wave period } \\ J & \text { Time between two consecutive turbulent bursts } \\ M & \text { Number of wave cycles for ensemble-averaging } \\ N & \text { Instantaneous velocity component (representing } u, v, \text { or } w) \\ R & \text { Sampling number in the time domain } \\ U & \text { Auto-correlation coefficients } \\ I_{1} & \text { Instantaneous streamwise velocity averaged across the } z \text {-axis } \\ I_{2} & \text { Total number of grid points in the } x \text { direction } \\ I_{3} & \text { Total number of grid points in the } y \text { direction } \\ & \text { Total number of grid points in the } z \text { direction } \\ \bar{M} & \end{array}$

\section{Acknowledgements}

The authors gratefully appreciate for the support from the UCL Dean's Prize and the China Scholarship Council (CSC).

\section{References}

1. Achia, B.U. and Thompson, D.W., 1976. Structure of the turbulent boundary in dragreducing pipe flow. Journal of Fluid Mechanics, 81(3), 439-464.

2. Adrian, R.J., Meinhart, C.D., Tomkins, C.D., 2000. Vortex organization in the outer region of the turbulent boundary layer. Journal of Fluid Mechanics, 422, 1-54.

3. Adrian, R.J. and Liu, Z.C., 2002. Observation of vortex packets in direct numerical simulation of fully turbulent channel flow. Journal of visualization, 5(1), 9-19.

4. Arnskov, M.M., Fredsøe, J. and Sumer, B.M., 1993. Bed shear stress measurements over a smooth bed in three-dimensional wave-current motion. Coastal Engineering, Vol. 20, pp. 277-316.

5. Bakker, W., and van Doorn, T., 1978. Near-bottom velocities in waves with a current. Proc. 16th Coastal Engng. Conf., Hamborg, ASCE, chapter 82, 1394-1413.

6. Burr, I.W., 1942. Cumulative frequency functions. The Annals of mathematical statistics, 13(2), 215-232.

7. Carstensen, S., Sumer, B.M. and Fredsøe, J., 2010. Coherent structures in wave boundary layers. Part 1. Oscillatory motion. Journal of Fluid Mechanics, Volume 646, pp. 169-206. 
8. Carstensen, S., Sumer, B.M. and Fredsøe, J., 2012. A note on turbulent spots over a rough bed in wave boundary layers. Physics of Fluids, Volume 24, pp. 115104-1 115104-13.

9. Christoffersen, J.B. and Jonsson, I.G., 1985. Bed friction and dissipation in a combined current and wave motion. Ocean Engineering, 12, 387-423.

10. Davies, A.G., Soulsby, R.L., and King, H.L., 1988. A numerical model of the combined wave and current bottom boundary layer. Journal of Geophysical Research, 93, 491-508.

11. Dennis, D.J., 2015. Coherent structures in wall-bounded turbulence. Anais da Academia Brasileira de Ciências, 87(2), 1161-1193.

12. Fredsøe, J., 1984. Turbulent boundary layer in wave-current motion. Journal of Hydraulic Engineering, 110, 1103-1120.

13. Fredsøe, J., Andersen, K.H., Sumer, B.M., 1999. Wave plus current over a ripplecovered bed. Coastal Engineering, 38, 177-221.

14. Galleno, M., O'Doherty, N. and Simons, R.R., 2002. Experimental and theoretical assessment of novel wave absorbers. In Coastal Engineering 2002: Solving Coastal Conundrums, 1353-1363.

15. Grant, W.D. and Madsen, O.S. 1979. Combined Wave and Current Interaction with a Rough Bottom. Journal of Geophysical Research, 84, 1797-1808.

16. Grass, A.J., 1971. Structural features of turbulent flow over smooth and rough boundaries. Journal of Fluid Mechanics, 50, 233-255.

17. Grass, A.J., Stuart, R.J., Mansour-Tehrani, M., 1991. Vortical Structures and Coherent Motion in Turbulent Flow over Smooth and Rough Boundaries. Physical Sciences and Engineering, 336, 35-65.

18. Grass, A.J., Stuart, R. J., Mansour-Tehrani, M., 1993. Common vortical structure of turbulent flows over smooth and rough boundaries. AIAA Journal, 31, 837-847.

19. Gupta, A.K., Laufer, J. and Kaplan, R.E., 1971. Spatial structure in the viscous sublayer. Journal of Fluid Mechanics, 50(3), 493-512.

20. Gyr, A. and Schmid, A., 1997. Turbulent flows over smooth erodible sand beds in flumes. Journal of hydraulic research, 35(4), 525-544.

21. Heathershaw, A.D. and Thorne, P.D., 1985. Sea-bed noises reveal role of turbulent bursting phenomenon in sediment transport by tidal currents. Nature, 316(6026), 339.

22. Hino, M., Kashiwayanagi, M., Nakayama, A. and Hara, T., 1983. Experiments on the turbulence statistics and the structure of a reciprocating oscillatory flow. Journal of Fluid Mechanics, 131, 363-400.

23. Holmedal, L.E., Myrhaug, D., Rue, H., 2003. The sea bed boundary layer under random waves plus current. Continental Shelf Research, 23, 717-750.

24. Jiménez, J. and Kawahara, G., 2013. Dynamics of wall-bounded turbulence. Ten Chapters in Turbulence. 221-268.

25. Johnson, R.W., 1998. The Handbook of Fluid Dynamics. Springer.

26. Kemp, P. H. and Simons, R. R. 1982. The interaction between waves and a turbulent current: waves propagating with the current. Journal of Fluid Mechanics, 116, 227250.

27. Kemp, P. H. and Simons, R. R. 1983. The interaction of waves and a turbulent current: waves propagating against the current. Journal of Fluid Mechanics, 130, 73-89.

28. Keylock, C.J., Lane, S.N. and Richards, K.S., 2014. Quadrant/octant sequencing and the role of coherent structures in bed load sediment entrainment. Journal of Geophysical Research: Earth Surface, 119(2), 264-286.

29. Kim, T.I., 1984. Mass transport in laboratory water wave flumes. Ph.D. Thesis, Oregon State University. 
30. Kim, H.T., Kline, S.J., and Reynolds, W.C., 1971. The production of turbulence near a smooth wall in a turbulent boundary layer. Journal of Fluid Mechanics, 50, 133-160.

31. Kline, S.J., Reynolds, W.C., Schraub, F.A., and Runstadler, P.W., 1967. The structure of turbulent boundary layers. Journal of Fluid Mechanics, 30, 741-773.

32. Klopman, G., 1994. Vertical structure of the flow due to waves and currents: laserDoppler flow measurements for waves following or opposing a current. Tech. Rep. Delft Hydraulics H840.32.

33. Kolmogorov, A.N., 1941. The local structure of turbulence in incompressible viscous fluid for very large Reynolds numbers. Proceedings of the USSR Academy of Sciences (in Russian), 30: 299-303.

34. Kravchenko, A.G., Choi, H. and Moin, P., 1993. On the relation of near-wall streamwise vortices to wall skin friction in turbulent boundary layers. Physics of Fluids A: Fluid Dynamics, 5(12), 3307-3309.

35. Lagraa, B., Labraga, L. and Mazouz, A., 2004. Characterization of low-speed streaks in the near-wall region of a turbulent boundary layer. European Journal of MechanicsB/Fluids, 23(4), 587-599.

36. Lamb, H., 1932. Hydrodynamics, 6th edition, Cambridge University Press, U.K.

37. Lee, M.K., Eckelman, L.D. and Hanratty, T.J., 1974. Identification of turbulent wall eddies through the phase relation of the components of the fluctuating velocity gradient. Journal of Fluid Mechanics, 66(1), 17-33.

38. Lelouvetel, J., Bigillon, F., Doppler, D., Vinkovic, I. and Champagne, J.Y., 2009. Experimental investigation of ejections and sweeps involved in particle suspension. Water resources research, 45(2).

39. Lodahl, C.R., Sumer, B.M., and Fredsøe, J., 1998. Turbulent combined oscillatory flow and current in a pipe. Journal of Fluid Mechanics, vol. 373, pp. 313-348.

40. Lu, S.S. and Willmarth, W.W., 1973. Measurements of the structure of the Reynolds stress in a turbulent boundary layer. Journal of Fluid Mechanics, 60(3), 481-511.

41. Lundgren, H., 1972. Turbulent currents in the presence of waves. Proc. 13th Coastal Engng. Conf., Vancouver, ASCE, chapter 33, 623-634.

42. Malarkey, J., Davies, A.G., 1998. Modelling wave-current interactions in rough turbulent bottom boundary layers. Ocean Engineering, 25, 119-141.

43. Mansour-Tehrani, M., 1992. Spatial distribution and scaling of bursting events in boundary layer turbulence over smooth and rough surfaces. Ph.D. Thesis, University of London.

44. Myrhaug, D., 1982. On a theoretical model of rough turbulent wave boundary layers. Ocean Engineering, 9(6), 547-565.

45. Myrhaug, D. and Slaattelid, O.H., 1989. Combined wave and current boundary layer model for fixed rough seabeds. Ocean engineering, 16(2), 119-142.

46. Nakagawa, H. and Nezu, I., 1981. Structure of space-time correlations of bursting phenomena in an open-channel flow. Journal of Fluid Mechanics, 104, 1-43.

47. Nelson, J.M., Shreve, R.L., McLean, S.R. and Drake, T.G., 1995. Role of near-bed turbulence structure in bed load transport and bed form mechanics. Water resources research, 31(8), 2071-2086.

48. Nielsen, P., 1992. Coastal bottom boundary layers and sediment transport, 4, World Scientific Publishing Co. Pte. Ltd., Singapore.

49. Nino, Y. and Garcia, M.H., 1996. Experiments on particle-turbulent interactions in the near wall region of an open channel flow: implications for sediment transport. J. Fluid Mech., vol. 326, p. 285.

50. Oldaker, D.K. and Tiederman, W.G., 1977. Spatial structure of the viscous sublayer in drag-reducing channel flows. The Physics of Fluids, 20(10), S133-S144. 
51. Pope, S.B., 2000. Turbulent flows. Cambridge University Press, Cambridge, U.K.

52. Ramaprian, B.R. \& Tu, S.W., 1983. Fully developed periodic turbulent pipe flow. Part 2. The detailed structure of the flow. J. Fluid Mech., 137, 59-81.

53. Ramsden, J.D. and Nath, J.H., 1989. Kinematics and return flow in a closed wave flume. Proc. 21st Coastal Engineering Conf., 448-462.

54. Robinson, S.K., 1991. Coherent motions in the turbulent boundary layer. Annual of Review of Fluid Mechanics, 23, 601-639.

55. Scandura, P., Faraci, C. \& Foti, E., 2016. A numerical investigation of accelerationskewed oscillatory flows, J. Fluid Mech., 808, pp.576-613.

56. Schoppa, W. and Hussain, F., 2002. Coherent structure generation in near-wall turbulence. Journal of fluid Mechanics, 453, 57-108.

57. Schraub, F.A. and Kline, S.J., 1965. Department of Mechanical Engineering, Stanford University. Rep. Md-12.

58. Simons, R.R., Grass, T.J., Tehrani, M.M., 1992. Bottom shear stresses in the boundary layers under waves and currents crossing at right angles. Coastal Engineering, chapter 45, 604-617.

59. Simons, R.R., Grass, T.J., Saleh, W.M., Tehrani, M.M., 1994. Bottom shear stresses under random waves with a current superimposed. Coastal Engineering, chapter 42, 565-578.

60. Simons, R.R., Myrhaug, D., Thais, L., Chapalain,G., Holmedal, L.E., and Maclver, R., 2000. Bed friction in combined wave-current flows. Coastal Engineering, 216-226.

61. Simons, R.R. and MacIver, R.D., 2001. Regular, bichromatic and random waves on co-linear currents. In: Hanson, $\mathrm{H}$ and Larson, M, (eds.) (Proceedings) 4th International Conference on Coastal Dynamics. (pp. 132-141). AMER SOC CIVIL ENGINEERS.

62. Sleath, J.F.A., 1984. Sea bed mechanics. John Wiley \& Sons Press, New York, U.S.

63. Smith, C. R., and Metzler, S. P., 1983. The characteristics of low-speed streaks in the near-wall region of a turbulent boundary layer. Journal of Fluid Mechanics, 129, 2754.

64. Soulsby, R.L., Hamm, L., Klopman, G., Myrhaug, D., Simons, R.R., and Thomas, G.P. 1993. Wave-current interaction within and outside the bottom boundary layer. Coastal Engineering, 21, 41-69.

65. Stretch, D.D., 1990. Automated pattern eduction from turbulent flow diagnostics. Center for Turbulence Research Annual Research Briefs, 145-157.

66. Sumer, B.M. and Oguz, B., 1978. Particle motions near the bottom in turbulent flow in an open channel. Journal of Fluid Mechanics, Vol. 86, pp. 109.

67. Sumer, B.M. and Deigaard, R., 1981. Particle motions near the bottom in turbulent flow in an open channel - Part 2. Journal of Fluid Mechanics, Vol. 109, pp. 311.

68. Sutherland, A.J., 1967. Proposed mechanism for sediment entrainment by turbulent flows. Journal of Geophysical Research, 72(24), 6183-6194.

69. Tadikamalla, P.R., 1980. A look at the Burr and related distributions. International Statistical Review, 48, 337-344.

70. Taylor, G.I., 1938. The spectrum of turbulence. Proceedings of the Royal Society of London. Series A, Mathematical and Physical Sciences, 476-490.

71. Teles, M. J., Pires-Silva, A., and Benoit, M., 2012. The influence of the turbulence closure model on wave-current interaction modelling at a local scale. Proceedings of 33rd Conference on Coastal Engineering, Santander, Spain, 1-12.

72. Teles, M.J., Pires-Silva, A.A., Benoit, M., 2013. Numerical modelling of wave current interactions at a local scale. Ocean Modelling, 68, 72-87. 
73. Umeyama, M., 2005. Reynolds Stresses and Velocity Distributions in a Wave-Current Coexisting Environment. Journal of Waterway, Port, Coastal, and Ocean engineering, 131, 203-212.

74. Umeyama, M., 2009. Changes in Turbulent Flow Structure under Combined WaveCurrent Motions. Journal of Waterway, Port, Coastal, and Ocean Engineering, 135, 213-227.

75. Umeyama, M., 2011. Coupled PIV and PTV Measurements of Particle Velocities and Trajectories for Surface Waves Following a Steady Current. Journal of Waterway, Port, Coastal, and Ocean Engineering, 137, 85-94.

76. Van der A, D.A., Scandura, P. \& O’Donoghue, T., 2018. Turbulence statistics in smooth wall oscillatory boundary layer flow, J. Fluid Mech., 849, pp.192-230.

77. Van Doorn, T., 1981. Experimental investigation of near bottom velocities in water waves with and without a current. Delft Hydraulics Laboratory, Tow-Report 1423.

78. You, Z.J., 1994a. A simple model for current velocity profiles in combined wavecurrent flows. Coastal Engineering, 23(3-4), 289-304.

79. You, Z.J., 1994b. Eddy viscosities and velocities in combined wave-current flows. Ocean engineering, 21(1), 81-97.

80. You, Z.J., Wilkinson, D.L. and Nielsen, P., 1991. Velocity distributions of waves and currents in the combined flow. Coastal Engineering, 15(5-6), 525-543.

81. You, Z.J., Wilkinson, D.L., and Nielsen, P., 1992. Velocity distribution in turbulent oscillatory boundary layer. Coastal engineering, 18, 21-38.

82. Yuan, J. and Madsen, O.S., 2014. Experimental study of turbulent oscillatory boundary layers in an oscillating water tunnel. Coastal Engineering, 89, 63-84.

83. Yuan, J. and Madsen, O.S., 2015. Experimental and theoretical study of wave-current turbulent boundary layers. Journal of Fluid Mechanics, 765, 480-523.

84. Zhang, J.S., Zhang, Y., Jeng, D.S., Liu, P.L.F., Zhang, C. 2014. Numerical simulation of wave-current interaction using a RANS solver. Ocean Engineering, 75, 157-164.

85. Zhang, X., Simons, R. and Buldakov, E., 2017. A numerical study of wave-current interaction in the bottom boundary layer. In Proceedings of 35th International Conference on Coastal Engineering, 35. 